Interfaces and Free Boundaries 14 (2012), 569-602

DOI 10.4171/IFB/292

\title{
Free boundary problems of magnetohydrodynamics in multi-connected domains
}

\author{
VSEVOLOD A. SOLONNIKOV \\ V.A. Steklov Mathematical Institute of the Russian Academy of Sciences, 197198 St. Petersburg, \\ Fontanka 27 \\ E-mail: solonnik@pdmi.ras.ru
}

[Received 15 February 2012 and in revised form 7 August 2012]

\begin{abstract}
The subject of the paper is the solvability theory of evolution free boundary problems of magnetohydrodynamics for viscous incompressible liquid in multi-connected domains. The main result is the local existence theorem for such problems under rather general assumptions on the initial data of the problem, i.e., on the initial configuration of the liquid and on the initial values of the velocity vector field $\boldsymbol{v}$ and of the magnetic field $\boldsymbol{H}$. The solution is found in anisotropic SobolevSlobodetskii spaces.
\end{abstract}

2010 Mathematics Subject Classification: Primary 35Q35, 76W05.

Keywords: Sobolev spaces, coercive estimates.

Dedicated to the memory of Prof. M. Padula and Prof. A. Lascu

\section{Formulation of the problem}

We consider the problem of motion of a viscous incompressible capillary electrically conducting liquid with a free surface surrounded with a vacuum. The liquid is subject to the mass forces, capillary force at the free boundary and forces generated by the magnetic field. This field is induced by the electric current prescribed in a fixed domain separated from the domain occupied with the liquid.

The governing equations are the Navier-Stokes equations with the additional terms containing the magnetic field and the Maxwell equations in quasistationary approximation, i.e., without the displacement current (see $[1,2])$.

Evolution initial-boundary value problems of magnetohydrodynamics are intensively studied in the mathematical literature (see, for instance, [3-17]). The major part of the papers deals with the problems in fixed domains. It seems that the first rigorous proof of the solvability of such problems is given in the paper [23], where a global existence of a weak solution of the Hopf's type and a local existence of a weak solution with the square integrable time derivative is established (moreover, it was proved that in the two-dimensional case this solution is global). These results are analogous to those of Kiselev-Ladyzhenskaya [18] and Ladyzhenskaya [19] for the Navier-Stokes equations. It has to be emphasized that in some problems studied in [3] the magnetic and electric fields are sought not only in the domain $\Omega_{1}$ filled with the liquid but also outside $\Omega_{1}$, in a surrounding vacuum region $\Omega_{2}$, where the governing equations are of a completely different type in comparison with $\Omega_{1}$.

The construction of the strong solution possessing square integrable second space derivatives of the velocity of the liquid $\boldsymbol{v}(x, t)$ and of the magnetic field $\boldsymbol{H}$ is carried out in [4] in the case of simply connected and in [17] for multi-connected $\Omega_{i}$. In particular, it is shown that in both cases 
the whole problem can be written in the form of the equation

$$
\boldsymbol{U}_{t}+Q \boldsymbol{U}+B \boldsymbol{U}=F,\left.\quad \boldsymbol{U}\right|_{t=0}=\boldsymbol{U}_{0}
$$

for $\boldsymbol{U}=(\boldsymbol{v}, \boldsymbol{H})$, where $Q$ is a linear positive self-adjoint operator in a Hilbert space and $B$ is a nonlinear operator (like in the case of the Navier-Stokes equations). This provides the possibility of studying the problems of magnetohydrodynamics with the methods of the semi-groups theory.

As for the free boundary problem, it has been first considered, to our knowledge, in the paper [20] in the case of simply connected $\Omega$. In the present paper the last condition is removed, which has required an essential change of the scheme of the analysis. This concerns the way of determination of the field $\boldsymbol{H}$, where the ideas of the paper [17] are used, whereas [20] was essentially based on the paper [4]. Using the transformation of independent variables that now is connected with the name of Hanzawa [21] (although it has been used earlier), we write the problem in a fixed domain with a smooth boundary, and we eliminate the electric field $\boldsymbol{E}$. The resulting nonlinear problem is solved in a certain finite time interval, on the basis of the coercive estimates for the solutions of the corresponding linearized problem, after which the construction of $\boldsymbol{E}$ is carried out (this step is missing in [20]). It should be also emphasized that one of the boundary conditions, namely, the jump condition on the free surface for the tangential part of $\boldsymbol{E}$, differs from the standard condition $\left[\boldsymbol{E}_{\tau}\right]=0$ on fixed surfaces, see (1.7), and this is essentially used in the construction of $\boldsymbol{E}$. At this point, a valuable help was delivered to the author by Dr. N. Filonov, who gave the proof of the important Proposition 1. The author brings him his deep gratitude. The author is indebted also to Prof. J. Rodrigues and to the referee for their important suggestions concerning the presentation of the paper.

Now we pass to the precise formulation of our problem. We assume that the liquid fills a variable connected compact domain $\Omega_{1 t} \subset \mathbb{R}^{3}$ whose boundary $\Gamma_{t}$ is a free surface. The liquid is subject to the external mass forces $\boldsymbol{f}(x, t)$, capillary forces on $\Gamma_{t}$ and forces arising due to the presence of the magnetic field. Moreover, in a fixed domain $\Omega_{3}$ an electric current of density $\boldsymbol{j}(x, t)$ is given. This vector field should have zero normal component on $\partial \Omega_{3}$ :

$$
\boldsymbol{j} \cdot \boldsymbol{n}=0
$$

and vanish outside $\Omega_{3}$.

Both $\Omega_{1 t}$ and $\Omega_{3}$ are surrounded by a vacuum region $\Omega_{2 t}$. The domain $\Omega=\bar{\Omega}_{1 t} \cup \bar{\Omega}_{3} \cup \Omega_{2 t}$ is bounded by a compact perfectly conducting surface $S$. If is assumed that the domains $\Omega_{1 t}$ and $\Omega_{3}$ are disjoint, as well as the surfaces $S$ and $\Gamma_{t}$ (see Fig. 1).

The case $S=\emptyset, \Omega=\mathbb{R}^{3}$ is not excluded.

The state of the medium in $\Omega$ is characterized by the following functions:

- $\boldsymbol{v}(x, t)$ : the velocity vector field of the liquid,

- $p(x, t)$ : the pressure function,

- $\boldsymbol{H}(x, t), x \in \Omega$ : the magnetic intensity,

- $\boldsymbol{B}(x, t)$ : the magnetic induction,

- $\boldsymbol{E}(x, t), x \in \Omega$ : the electric intensity.

The vector fields $\boldsymbol{H}(x, t)$ and $\boldsymbol{B}(x, t)$ are connected by the relation

$$
\boldsymbol{B}(x, t)=\mu \boldsymbol{H}(x, t)
$$

where $\mu$ is a piece-wise constant function of magnetic permeability taking positive values $\mu_{1}$ in $\Omega_{1 t}, \mu_{2}$ in $\Omega_{2 t}, \mu_{3}$ in $\Omega_{3}$. The domain $\Omega_{3}$ may consist of several components with different values of $\mu_{3}$. 


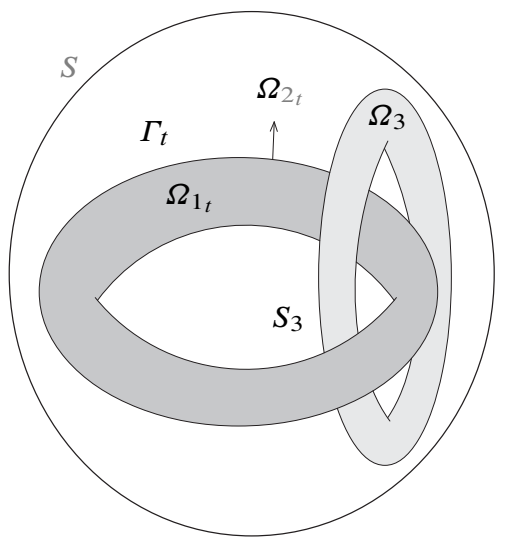

FIG. 1.

The motion of the liquid is governed by the Navier-Stokes equations

$$
\left\{\begin{array}{l}
\boldsymbol{v}_{t}+(\boldsymbol{v} \cdot \nabla) \boldsymbol{v}-\nabla \cdot T(\boldsymbol{v}, p)-\nabla \cdot T_{M}(\boldsymbol{H})=\boldsymbol{f}(x, t), \\
\nabla \cdot \boldsymbol{v}(x, t)=0, \quad x \in \Omega_{1 t}, \quad t>0
\end{array}\right.
$$

where

- $T(\boldsymbol{v}, p)=-p I+v S(v)$ is the viscous stress tensor,

- $S(\boldsymbol{v})=\nabla v+(\nabla v)^{T}$ is the doubled rate-of-strain tensor,

- $T_{M}(\boldsymbol{H})=\mu\left(\boldsymbol{H} \otimes \boldsymbol{H}-\frac{1}{2}|\boldsymbol{H}|^{2} I\right)$ is the magnetic stress tensor.

Electric and magnetic fields satisfy the system of the Maxwell equations with the omitted displacement current

$$
\left\{\begin{array}{l}
\mu \boldsymbol{H}_{t}=-\operatorname{rot} \boldsymbol{E}, \quad \nabla \cdot \boldsymbol{H}=0, \quad x \in \Omega_{1 t} \cup \Omega_{2 t} \cup \Omega_{3}, \\
\operatorname{rot} \boldsymbol{H}=\alpha(\boldsymbol{E}+\mu(\boldsymbol{v} \times \boldsymbol{H})), \quad x \in \Omega_{1 t}, \quad t>0, \\
\operatorname{rot} \boldsymbol{H}=\alpha \boldsymbol{E}+\boldsymbol{j}(x, t), \quad x \in \Omega_{3}, \\
\operatorname{rot} \boldsymbol{H}=0, \quad \nabla \cdot \boldsymbol{H}=0, \quad \nabla \cdot \boldsymbol{E}=0, \quad x \in \Omega_{2 t} .
\end{array}\right.
$$

where $\alpha$ is a piece-wise constant function of conductivity, positive for $x \in \Omega_{1 t} \cup \Omega_{3}$ and equal to zero in $\Omega_{2 t}$. The last equation for $\boldsymbol{E}$ follows from the fact that the vacuum region $\Omega_{2 t}$ can not contain electric charges.

Let us turn to the boundary conditions on the exterior boundary $S$ and on the interfaces $\Gamma_{t}$ and $S_{3}$ separating media with different physical properties. On fixed surfaces $S_{3}=\partial \Omega_{3}$ and $S$ standard boundary and jump conditions are prescribed (see $[1,2])$ :

$$
\left\{\begin{array}{l}
\boldsymbol{H} \cdot \boldsymbol{n}=0, \quad \boldsymbol{E}_{\tau}=0, \quad x \in S, \\
{[\mu \boldsymbol{H} \cdot \boldsymbol{n}]=0, \quad\left[\boldsymbol{H}_{\tau}\right]=0, \quad\left[\boldsymbol{E}_{\tau}\right]=0, \quad x \in S_{3},}
\end{array}\right.
$$

and on the free surface $\Gamma_{t}$ we have

$$
\left\{\begin{array}{l}
\left(T(\boldsymbol{v}, p)+\left[T_{M}(\boldsymbol{H})\right]\right) \boldsymbol{n}=\sigma \boldsymbol{n} H, \quad V_{n}=\boldsymbol{v} \cdot \boldsymbol{n}, \\
{[\mu \boldsymbol{H} \cdot \boldsymbol{n}]=0, \quad\left[\boldsymbol{H}_{\tau}\right]=0}
\end{array}\right.
$$


where

- $H$ is the doubled mean curvature of $\Gamma_{t}$,

- $\boldsymbol{H}_{\tau}=\boldsymbol{H}-\boldsymbol{n}(\boldsymbol{n} \cdot \boldsymbol{H}), \boldsymbol{E}_{\tau}=\boldsymbol{E}-\boldsymbol{n}(\boldsymbol{n} \cdot \boldsymbol{E})$ are tangential components of $\boldsymbol{H}$ and $\boldsymbol{E}$,

- $[\boldsymbol{F}]$ is a jump of the vector field $\boldsymbol{F}(x), x \in \Omega_{1 t} \cup \Omega_{2 t} \cup \Omega_{3}$, on $\Gamma_{t}$ and $S_{3}$,

- $V_{n}$ is the velocity of evolution of $\Gamma_{t}$ in the direction of the exterior normal $\boldsymbol{n}$.

It remains to write the jump condition for $\boldsymbol{E}_{\tau}$ on $\Gamma_{t}$. Usually the jump conditions are deduced from the Maxwell equations, assuming that they are satisfied in the sense of the distribution theory. For instance, from

$$
\int_{K} \boldsymbol{B} \cdot \nabla \varphi(x) d x=0
$$

where $\varphi \in C_{0}^{\infty}(K), K \subset \Omega, K \cup \Gamma_{t} \neq \emptyset$ it follows that

$$
-\int_{K} \nabla \cdot \boldsymbol{B} \varphi d x+\int_{\Gamma_{t} \cap K}[\boldsymbol{B} \cdot \boldsymbol{n}] \varphi d S=0,
$$

and this implies

$$
\nabla \cdot \boldsymbol{B}=0, \quad x \in K \backslash \Gamma_{t}, \quad[\boldsymbol{B} \cdot \boldsymbol{n}]=0, \quad x \in \Gamma_{t} \cap K .
$$

In the same way the jump condition $\left.\left[\boldsymbol{H}_{\tau}\right]\right|_{\Gamma_{t}}=0$ is deduced. Moreover, if the equation $\boldsymbol{B}_{t}=$ $-\operatorname{rot} \boldsymbol{E}$ is satisfied in a generalized sense, i.e.,

$$
\int_{t_{0}}^{t_{0}+\tau} \int_{K}\left(-\boldsymbol{B} \cdot \boldsymbol{\varphi}_{t}+\boldsymbol{E} \cdot \operatorname{rot} \boldsymbol{\varphi}\right) d x d t=0,
$$

then on the manifold $\mathfrak{G}_{\tau}=\left\{x \in \Gamma_{t}, t \in\left(t_{0}, t_{0}+\tau\right)\right\} \subset \mathbb{R}^{4}$ the relation

$$
\mathfrak{n}_{t}\left[\boldsymbol{B}_{\tau}\right]+\left[\mathfrak{n}_{x} \times \boldsymbol{E}\right]=0
$$

holds, where $\mathfrak{n}_{x}=\left(\mathfrak{n}_{1}, \mathfrak{n}_{2}, \mathfrak{n}_{3}\right)$ and $\mathfrak{n}_{t}$ are components of the normal vector $\mathfrak{n}$ to $\mathfrak{G}_{\tau}$ in $\mathbb{R}^{4}$. In particular, for fixed interfaces, when $\mathfrak{n}_{t}=0$, the condition (1.7) takes a standard form $\left[\mathfrak{n}_{x} \times \boldsymbol{E}\right]=0$, i.e., $\left[\boldsymbol{E}_{\tau}\right]=0$.

The following proposition is important for the construction of the solution of (1.2)-(1.5), (1.7).

Proposition 1 If the relations (1.6) and (1.7) are satisfied, then

$$
[\boldsymbol{n} \cdot \boldsymbol{r} \text { ot } \boldsymbol{E}]=-\left[\boldsymbol{n} \cdot \boldsymbol{B}_{t}\right], \quad x \in \Gamma_{t} .
$$

Proof. Suppose the surface $\mathfrak{G}_{\tau} \subset K \times\left(t_{0}, t_{0}+\tau\right) \subset \mathbb{R}^{4}$ is given by the equation $\xi_{3}=z\left(\xi_{1}, \xi_{2}, t\right)$, where $\left(\xi_{1}, \xi_{2}, \xi_{3}\right)$ are Cartesian coordinates in $\mathbb{R}^{3}$. The functions given on $\mathfrak{G}_{\tau}$ can be considered as functions of $\xi_{1}, \xi_{2}, t$. The normal $\mathfrak{n}$ to $\mathfrak{G}_{\tau}$ is parallel to the vector $\left.\widetilde{\boldsymbol{n}}, z_{t}\right)$, where $\widetilde{\boldsymbol{n}}=\left(z_{\xi_{1}}, z_{\xi_{2}},-1\right)$.

It is easily verified that

$$
\begin{aligned}
-[\widetilde{\boldsymbol{n}} \cdot \boldsymbol{r} \text { ot } \boldsymbol{E}] & =\left[z_{\xi_{1}}\left(E_{2,3}-E_{3,2}\right)+z_{\xi_{2}}\left(E_{3,1}-E_{1,3}\right)-\left(E_{1,2}-E_{2,1}\right)\right] \\
& =\left[\frac{\partial}{\partial \xi_{1}}\left(z_{\xi_{2}} E_{3}+E_{2}\right)+\frac{\partial}{\partial \xi_{2}}\left(-E_{1}-z_{\xi_{1}} E_{3}\right)\right] \\
& =\frac{\partial}{\partial \xi_{1}}[\widetilde{\boldsymbol{n}} \times \boldsymbol{E}]_{1}+\frac{\partial}{\partial \xi_{2}}[\widetilde{\boldsymbol{n}} \times \boldsymbol{E}]_{2},
\end{aligned}
$$


where $E_{i, j}$ is the partial derivative of $E_{i}$ with respect to $\xi_{j}$ and

$$
\frac{\partial \boldsymbol{E}}{\partial \xi_{\alpha}}=\boldsymbol{E}_{, \alpha}+z_{\xi_{\alpha}} \boldsymbol{E}_{, 3}, \quad \alpha=1,2,
$$

are derivatives calculated taking into account the dependence of $\boldsymbol{E}$ on $\xi_{3}$. By (1.7), the equation (1.9) is equivalent to

$$
\begin{aligned}
-[\widetilde{\boldsymbol{n}} \cdot \boldsymbol{r o t} \boldsymbol{E}] & =-\frac{\partial}{\partial \xi_{1}} z_{t}\left[B_{1}\right]-\frac{\partial}{\partial \xi_{2}} z_{t}\left[B_{2}\right] \\
& =-\left[z_{\xi_{1}, t} B_{1}+z_{t} B_{1,1}+z_{\xi_{1}} z_{t} B_{1,3}+z_{\xi_{2}, t} B_{2}+z_{t} B_{2,2}+z_{\xi_{2}} z_{t} B_{2,3}\right] .
\end{aligned}
$$

Now we differentiate $[\widetilde{\boldsymbol{n}} \cdot \boldsymbol{B}]=0$ with respect to $t$, which leads to

$$
\left[z_{\xi_{1}, t} B_{1}+z_{\xi_{1}} B_{1, t}+z_{t} z_{\xi_{1}} B_{1,3}+z_{\xi_{2}, t} B_{2}+z_{\xi_{2}} B_{2, t}+z_{\xi_{2}} z_{t} B_{2,3}-B_{3, t}-z_{t} B_{3,3}\right]=0 .
$$

When we add (1.11) to (1.10) and take the equation $\nabla \cdot \boldsymbol{B}=0$ into account, we obtain

$$
-\widetilde{\boldsymbol{n}} \cdot \boldsymbol{r} \text { ot } \boldsymbol{E}]=\left[z_{\xi_{1}} B_{1, t}+z_{\xi_{2}} B_{2, t}-B_{3, t}\right]=\left[\widetilde{\boldsymbol{n}} \cdot \boldsymbol{B}_{t}\right],
$$

The proof of the Proposition is due to Dr. N. Filonov.

Let us go back to the formulation of the problem. At the initial moment $t=0$ the configuration of the liquid and the values of $\boldsymbol{v}(x, 0)$ and $\boldsymbol{H}(x, 0)$ are prescribed:

$$
\boldsymbol{v}(x, 0)=\boldsymbol{v}_{0}(x), \quad x \in \Omega_{10}, \quad \boldsymbol{H}(x, 0)=\boldsymbol{H}_{0}(x), \quad x \in \Omega_{10} \cup \Omega_{20} \cup \Omega_{3},
$$

where $\Omega_{10}, \Omega_{20}$ are given domains. Finally, we need some normalization conditions for $\boldsymbol{E}(x, t)$, $x \in \Omega_{2}$. Indeed, together with $\boldsymbol{E}$ the vector field $\boldsymbol{E}+\boldsymbol{E}^{\prime}$ also satisfies (1.3), (1.4), if $\boldsymbol{E}^{\prime}=0$ in $\Omega_{1 t} \cup \Omega_{3}$ and $\boldsymbol{E}^{\prime}$ is so called Dirichlet vector field in $\Omega_{2 t}$, satisfying the conditions

$$
\operatorname{rot} \boldsymbol{E}^{\prime}(x, t)=0, \quad \nabla \cdot \boldsymbol{E}^{\prime}(x, t)=0, \quad x \in \Omega_{2 t}, \quad \boldsymbol{E}_{\tau}^{\prime}=0, \quad x \in \partial \Omega_{2 t},
$$

in other words, $\boldsymbol{E}^{\prime}(x, t)=\sum_{j=1}^{b_{2}} C_{j}(t) \chi_{j}(x, t)$,

$$
\nabla^{2} \chi_{j}(x, t)=0, \quad x \in \Omega_{2 t},\left.\quad \chi_{j}(x, t)\right|_{x \in S_{k}}=\delta_{j k},\left.\quad \chi_{j}(x, t)\right|_{x \in \Gamma_{t}}=0,
$$

where $S_{k}, k=1, \ldots, b_{2}$ are all the connected components of $\partial \Omega_{2 t}$, except $\Gamma_{t}$ and $b_{2}$ is the second Betti number of $\Omega_{2 t}$. The normalization conditions can be taken in the form

$$
\int_{S_{k}} \boldsymbol{E} \cdot \boldsymbol{n} d S=0
$$

Since the matrix with the elements $\int_{S_{i}} \frac{\partial \chi_{j}}{\partial n} d S, i, j=1, \ldots, b_{2}$ is not degenerate, the equations (1.13) define $C_{j}(t)$ (and the vector field $\left.\boldsymbol{E}(x, t), x \in \Omega_{2 t}\right)$ in a unique way. 
The aim of the paper is to prove local (in time) unique solvability of the problem (1.2)-(1.5), (1.7), (1.12), (1.13) under the assumption that the initial data satisfy only natural compatibility conditions

$$
\begin{aligned}
& \nabla \cdot \boldsymbol{v}_{0}(x)=0, \quad x \in \Omega_{10}, \quad\left[\left(S\left(\boldsymbol{v}_{0}\right) \boldsymbol{n}\right)_{\tau}\right]=0, \quad x \in \Gamma_{0}, \\
& \nabla \cdot \boldsymbol{H}_{0}(x)=0, \quad x \in \Omega_{10} \cup \Omega_{20} \cup \Omega_{30}, \quad \operatorname{rot} \boldsymbol{H}_{0}(x)=0, \quad x \in \Omega_{20}, \\
& {\left[\boldsymbol{H}_{0 \tau}\right]=0, \quad\left[\mu \boldsymbol{H}_{0} \cdot \boldsymbol{n}_{0}\right]=0, \quad x \in \Gamma_{0},} \\
& {\left[\boldsymbol{H}_{0 \tau}\right]=0, \quad\left[\mu \boldsymbol{H}_{0} \cdot \boldsymbol{n}\right]=0, \quad x \in S_{3},} \\
& \boldsymbol{H}_{0}(x) \cdot \boldsymbol{n}=0, \quad x \in S,
\end{aligned}
$$

where $\boldsymbol{n}_{0}$ is the exterior normal to $\Gamma_{0}$.

The precise formulation of the result is given in Theorem 2.1.

\section{Transformation of the problem and formulation of the main result}

It is customary to write free boundary problems as nonlinear problems in fixed domains. We introduce now the corresponding coordinate transformation. We assume that $\Gamma_{0}$ is located in the neighborhood of a smooth connected surface $\mathcal{G}$ of arbitrary topological type, and can be regarded as a normal perturbation of $\mathrm{G}$ :

$$
\Gamma_{0}=\left\{x=y+N(y) \rho_{0}(y), \quad y \in \mathcal{G}\right\}
$$

where $\rho_{0}$ is a given small function and $\boldsymbol{N}(y)$ is the exterior normal to 9 . Moreover, we assume that also for $t>0$

$$
\Gamma_{t}=\{x=y+N(y) \rho(y, t), \quad y \in \mathcal{G}\},
$$

with an unknown function $\rho(y, t)$ such that $\rho(y, 0)=\rho_{0}(y)$. We extend $N(y)$ and $\rho(y, t)$ from $\mathcal{G}$ into $\Omega$ in such a way that the extension $N^{*}$ of $N$ is a smooth non-zero regular function in $\Omega$ and $\rho^{*}$ vanishes in $\Omega_{3}$ and near $S \cup S_{3}$ and satisfies the inequalities (4.11) (hence $\rho^{*}$ is small for small $\rho$ ). We denote by $\mathcal{F}_{1}$ the domain bounded by $\mathcal{G}$ and we set $\mathcal{F}_{2}=\Omega \backslash\left(\mathcal{F}_{1} \cup \bar{\Omega}_{3}\right.$ ), $\Omega_{3} \equiv \mathcal{F}_{3}$ (for uniformity of notation).

The transformation

$$
x \equiv e_{\rho}(y, t)=y+N^{*}(y) \rho^{*}(y, t), \quad y \in \mathcal{G}
$$

maps $\mathcal{F}_{i}$ on $\Omega_{i t}, i=1,2$, and leaves $\Omega_{3}$ invariant. We denote by $\mathcal{L}=\mathcal{L}\left(y, \rho^{*}\right)=\left(l_{i j}\right)_{i, j=1,2,3}$ the Jacobi matrix of the transformation (2.2) and we set $J=\operatorname{det} \mathcal{L}, \widehat{\mathcal{L}}=J \mathcal{L}^{-1}=\left(\widehat{L}_{i j}\right)_{i, j=1,2,3} ; \widehat{\mathcal{L}}$ is the co-factors matrix of $\mathcal{L}$. We note that $J\left(y, \rho^{*}\right)=1$ and $\mathcal{L}=\widehat{\mathcal{L}}=I$ for $y \in \mathcal{F}_{3}$ and $y \in S$.

We set

$$
\boldsymbol{u}(y, t)=\boldsymbol{v}\left(e_{\rho}, t\right) \quad q(y, t)=p\left(e_{\rho}, t\right),
$$

introduce new unknown vector fields

$$
\boldsymbol{h}=\widehat{\mathcal{L}} \boldsymbol{H}\left(e_{\rho}, t\right), \quad \boldsymbol{e}=\widehat{\mathcal{L}} \boldsymbol{E}\left(e_{\rho}, t\right),
$$


and make use of the formulas

$$
\begin{gathered}
\boldsymbol{n}\left(e_{\rho}\right)=\frac{\widehat{\mathcal{L}}^{T}(y, \rho) \boldsymbol{N}(y)}{\left|\widehat{\mathcal{L}}^{T}(y, \rho) \boldsymbol{N}(y)\right|}, \quad y \in \mathcal{G}, \\
\nabla \cdot \boldsymbol{h}(y, t)=\widehat{\mathcal{L}}^{T} \nabla_{y} \cdot \boldsymbol{H}\left(e_{\rho}, t\right)=\left.J\left(y, \rho^{*}\right) \nabla_{x} \cdot \boldsymbol{H}(x, t)\right|_{x=e_{\rho}(y, t),} \\
\nabla \cdot \boldsymbol{e}(y, t)=\left.J\left(y, \rho^{*}\right) \nabla_{x} \cdot \boldsymbol{E}(x, t)\right|_{x=e_{\rho}(y, t)}, \\
\operatorname{rot}_{x} \boldsymbol{H}(x, t)=\frac{\mathcal{L}}{J} \operatorname{rot}_{y} \mathbb{P} \boldsymbol{h}(y, t), \quad \operatorname{rot}_{x} \boldsymbol{E}(x, t)=\frac{\mathcal{L}}{J} \operatorname{rot}_{y} \boldsymbol{P e}(y, t),
\end{gathered}
$$

where $x=e_{\rho}(y, t)$ and

$$
\mathcal{P}\left(y, \rho^{*}\right)=\frac{\mathcal{L}^{T}}{J} \mathcal{L} .
$$

Repeating the calculations from [20], Sec.1, we show that the transformation (2.2) converts (1.2)(1.5) in

$$
\begin{aligned}
& \left\{\begin{array}{l}
\boldsymbol{u}_{t}-\rho_{t}^{*}\left(\mathcal{L}^{-1} N^{*}(y) \cdot \nabla\right) \boldsymbol{u}+\left(\mathcal{L}^{-1} \boldsymbol{u} \cdot \nabla\right) \boldsymbol{u} \\
-\widetilde{\nabla} \cdot \widetilde{T}(\boldsymbol{u}, q)-\widetilde{\nabla} \cdot T_{M}\left(\frac{\mathcal{L}}{J} \boldsymbol{h}\right)=\boldsymbol{f}\left(e_{\rho}, t\right), \\
\nabla \cdot \widehat{\mathcal{L}} \boldsymbol{u}=0, \quad y \in \mathcal{F}_{1}, \quad t>0,
\end{array}\right. \\
& \left\{\begin{array}{l}
\mu\left(\boldsymbol{h}_{t}-\frac{1}{J} \widehat{\mathcal{L}}_{t} \mathcal{L} \boldsymbol{h}-\rho_{t}^{*} \widehat{\mathcal{L}}\left(\mathcal{L}^{-1} \boldsymbol{N}^{*}(y) \cdot \nabla\right) \frac{1}{J} \mathcal{L} \boldsymbol{h}\right)=-\operatorname{rot} \mathbb{P} \boldsymbol{e}, \quad y \in \mathcal{F}_{1} \cup \mathcal{F}_{2} \cup \mathcal{F}_{3}, \\
\operatorname{Prot} \mathbb{P} \boldsymbol{h}=\alpha\left(\mathbb{P} \boldsymbol{e}+\mu\left(\mathcal{L}^{-1} \boldsymbol{u} \times \boldsymbol{h}\right)\right), \quad \nabla \cdot \boldsymbol{h}=0, \quad y \in \mathcal{F}_{1}, \\
\operatorname{rot} \mathbb{P} \boldsymbol{h}=0, \quad \nabla \cdot \boldsymbol{h}=0, \quad \nabla \cdot \boldsymbol{e}=0, \quad x \in \mathcal{F}_{2}, \\
\operatorname{roth}=\alpha \boldsymbol{e}+\boldsymbol{j}(y, t), \quad \nabla \cdot \boldsymbol{h}=0, \quad y \in \mathcal{F}_{3},
\end{array}\right. \\
& \left\{\begin{array}{l}
\boldsymbol{h} \cdot \boldsymbol{n}=0, \quad \boldsymbol{e}_{\tau}=0, \quad y \in S, \\
{[\mu \boldsymbol{h} \cdot \boldsymbol{n}]=0, \quad\left[\boldsymbol{h}_{\tau}\right]=0, \quad\left[\boldsymbol{e}_{\tau}\right]=0, \quad y \in S_{3},}
\end{array}\right. \\
& \left\{\begin{array}{l}
{[\mu \boldsymbol{h} \cdot \boldsymbol{N}]=0, \quad\left[\boldsymbol{h}-\frac{\widehat{\mathcal{L}} \widehat{\mathcal{L}}^{T} \boldsymbol{N}}{\left|\hat{\mathcal{L}}^{T} \boldsymbol{N}\right|^{2}}(\boldsymbol{h} \cdot \boldsymbol{N})\right]=0,} \\
\widetilde{T}(\boldsymbol{u}, q) \boldsymbol{n}\left(e_{\rho}\right)+\left[T\left(\frac{\mathcal{L}}{J} \boldsymbol{h}\right) \boldsymbol{n}\left(e_{\rho}\right)\right]=\sigma H \boldsymbol{n}, \quad \rho_{t}=\frac{\boldsymbol{u} \cdot \widehat{\mathcal{L}}^{T} \boldsymbol{N}}{\Lambda(y, \rho)}, \quad y \in \mathcal{G},
\end{array}\right.
\end{aligned}
$$

where

- $\widetilde{\nabla}=\mathcal{L}^{-T} \nabla_{y}$ is the transformed gradient $\nabla_{x}$ ("T" means transposition, $\left.\mathcal{L}^{-T}=\left(\mathcal{L}^{-1}\right)^{T}\right)$;

- $\widetilde{S}(\boldsymbol{u})=\widetilde{\nabla} \boldsymbol{u}+(\widetilde{\nabla} \boldsymbol{u})^{T}$ is the transformed rate-of-strain tensor,

- $\widetilde{T}(\boldsymbol{u}, q)=-q I+v \widetilde{S}(\boldsymbol{u})$ is the transformed stress tensor,

- $\Lambda(y, \rho)=N(y) \cdot \widehat{\jmath}(y, \rho) N(y)=1-\rho \mathcal{H}_{G}(y)+\rho^{2} \mathcal{K}_{G}(y)$ and

- $\mathcal{H}_{\mathrm{g}}, \mathcal{K}_{\mathrm{g}}$ are the doubled mean curvature and the Gaussian curvature of $\mathrm{G}$, respectively.

Now we turn to the boundary condition (1.7). We compute the components $\mathfrak{n}_{t}$ and $\mathfrak{n}_{x}$ of the normal to the surface $\left\{x \in \Gamma_{t}, t \in(0, T)\right\}$ in $\mathbb{R}^{4}$. We consider the four-dimensional transformation $\left(x, t^{\prime}\right)=\mathfrak{e}_{\rho}(y, t)$ defined by

$$
x=e_{\rho}(y, t), \quad t^{\prime}=t
$$

and we make use of the four-dimensional analog of (2.4), i.e.,

$$
\mathfrak{n}\left(e_{\rho}, t\right)=\frac{\widehat{\mathfrak{L}}^{T} N_{0}}{\left|\widehat{\mathfrak{L}}^{T} N_{0}\right|},
$$


where $N_{0}(y)=\left(N_{1}(y), N_{2}(y), N_{3}(y), 0\right)$ is the normal to $\mathcal{G} \times(0, T)$ and $\widehat{\mathfrak{L}}$ is the co-factors matrix corresponding to the transformation (2.11). The Jacobi matrix of this transformation is given by

$$
\mathfrak{L}=\left(\begin{array}{cccc}
l_{11} & l_{12} & l_{13} & N_{1}^{*}(y) \rho_{t}^{*}(y, t) \\
l_{21} & l_{22} & l_{23} & N_{2}^{*}(y) \rho_{t}^{*}(y, t) \\
l_{31} & l_{32} & l_{33} & N_{3}^{*}(y) \rho_{t}^{*}(y, t) \\
0 & 0 & 0 & 1
\end{array}\right)
$$

hence

$$
\widehat{\mathfrak{L}}=\left(\begin{array}{cccc}
\widehat{L}_{11} & \widehat{L}_{12} & \widehat{L}_{13} & -(\widehat{\mathcal{L} N})_{1} \rho_{t}(y, t) \\
\widehat{L}_{21} & \widehat{L}_{22} & \widehat{L}_{23} & -(\widehat{\mathcal{L} N})_{2} \rho_{t}(y, t) \\
\widehat{L}_{31} & \widehat{L}_{32} & \widehat{L}_{33} & -(\widehat{\mathcal{L} N})_{3} \rho_{t}(y, t) \\
0 & 0 & 0 & J
\end{array}\right)
$$

for $y \in \mathcal{G}$ and

$$
\widehat{\mathfrak{L}}^{T} N_{0}=\left(\widehat{\mathscr{L}}^{T} N,-\Lambda \rho_{t}\right)
$$

It follows that (1.7) is equivalent to

$$
-\Lambda \rho_{t}[\mu \boldsymbol{H}]+\left[\widehat{\mathcal{L}}^{T} \boldsymbol{N} \times \boldsymbol{E}\right]=0, \quad x=e_{\rho}(y, t)
$$

or, in view of the algebraic identity $A \boldsymbol{f} \times A \boldsymbol{g}=\widehat{A}^{T}(\boldsymbol{f} \times \boldsymbol{g})$ and the kinematic boundary condition, to

$$
-\left(\boldsymbol{u} \cdot \widehat{\mathcal{L}}^{T} \boldsymbol{N}\right)[\mu \boldsymbol{h}]+J[\boldsymbol{N} \times \boldsymbol{P} \boldsymbol{e}]=0 .
$$

Finally, we have the initial and normalization conditions

$$
\left\{\begin{array}{l}
\boldsymbol{u}(y, 0)=\boldsymbol{u}_{0}(y)=\boldsymbol{v}_{0}\left(e_{\rho_{0}}\right), \quad y \in \mathcal{F}_{1}, \quad \rho(y, 0)=\rho_{0}(y), \quad y \in \mathcal{G}, \\
\boldsymbol{h}_{0}(y, 0)=\boldsymbol{h}_{0}(y)=\widehat{\mathcal{L}}\left(y, \rho_{0}^{*}\right) \boldsymbol{H}_{0}\left(e_{\rho_{0}}\right), \quad y \in \mathcal{F}_{1} \cup \mathcal{F}_{2} \cup \mathcal{F}_{3}, \\
\int_{S_{k}} \boldsymbol{e}(y, t) \cdot \boldsymbol{n}(y) d S=0, \quad k=1, \ldots, b_{2}\left(\mathfrak{F}_{2}\right) .
\end{array}\right.
$$

We find the solution of the problem (2.7)-(2.10), (2.13), (2.14) in anisotropic Sobolev-Slobodetskii spaces. We recall the definition of the corresponding norms. Let $\Omega$ be a domain in $\mathbb{R}^{n}$. The (isotropic) Sobolev space $W_{2}^{l}(\Omega)$ with $l>0$ is the space of functions $u(x), x \in \Omega$, with the norm

$$
\|u\|_{W_{2}^{l}(\Omega)}^{2}=\sum_{0 \leqslant|j| \leqslant l}\left\|D^{j} u\right\|_{L_{2}(\Omega)}^{2} \equiv \sum_{0 \leqslant|j| \leqslant l} \int_{\Omega}\left|D^{j} u(x)\right|^{2} d x,
$$

if $l=[l]$, i.e. $l$ is an integral number, and

$$
\|u\|_{W_{2}^{l}(\Omega)}^{2}=\|u\|_{W_{2}^{[l]}(\Omega)}^{2}+\sum_{|j|=[l]} \int_{\Omega} \int_{\Omega}\left|D^{j} u(x)-D^{j} u(y)\right|^{2} \frac{d x d y}{|x-y|^{n+2 \lambda}},
$$

if $l=[l]+\lambda, \lambda \in(0,1)$. As usual, $D^{j} u$ denotes a (generalized) partial derivative $\frac{\partial^{|j|} u}{\partial x_{1}^{j_{1}} \ldots \partial x_{n}^{j_{n}}}$ where $j=\left(j_{1}, j_{2}, \ldots, j_{n}\right)$ and $|j|=j_{1}+\ldots+j_{n}$. The anisotropic space $W_{2}^{l, l / 2}\left(Q_{T}\right), Q_{T}=\Omega \times(0, T)$, can be defined as

$$
L_{2}\left((0, T), W_{2}^{l}(\Omega)\right) \cap W_{2}^{l / 2}\left((0, T), L_{2}(\Omega)\right)
$$


and supplied with the norm

$$
\|u\|_{W_{2}^{l, l / 2}\left(Q_{T}\right)}^{2}=\int_{0}^{T}\|u(\cdot, t)\|_{W_{2}^{l}(\Omega)}^{2} d t+\int_{\Omega}\|u(x, \cdot)\|_{W_{2}^{l / 2}(0, T)}^{2} d x .
$$

There exist many other equivalent norms in $W_{2}^{l, l / 2}\left(Q_{T}\right)$; some of them will be used below. Sobolev spaces of functions given on smooth surfaces, in particular, on $\mathcal{G}$ and on $G_{T}=\mathcal{G} \times(0, T)$, are introduced in a standard way, with the help of local maps and partition of unity. We also find it convenient to introduce the spaces $W_{2}^{l, 0}\left(Q_{T}\right)=L_{2}\left(0, T ; W_{2}^{l}(\Omega)\right)$ and $W_{2}^{0, l / 2}\left(Q_{T}\right)=$ $W_{2}^{l / 2}\left(0, T ; L_{2}(\Omega)\right)$; the squares of norms in these spaces coincide, respectively, with the first and the second integral in (2.15).

In order to obtain uniform estimates of the solutions of the above problem for small $T$, we introduce in $W_{2}^{l, l / 2}\left(Q_{T}\right)$ equivalent norms defined by

$$
\|u\|_{\widehat{W}_{2}^{l, l / 2}\left(Q_{T}\right)}=\|u\|_{W_{2}^{l, l / 2}\left(Q_{T}\right)},
$$

if $l / 2$ is an integer or $l / 2=[l / 2]+\lambda, \lambda \in(1 / 2,1)$,

$$
\|u\|_{\widehat{W}_{2}^{l, l / 2}\left(Q_{T}\right)}^{2}=\|u\|_{W_{2}^{l, l / 2}\left(Q_{T}\right)}^{2}+\frac{1}{T^{2 \lambda}}\left\|\frac{\partial^{[l / 2]}}{\partial t^{[l / 2]}} u\right\|_{L_{2}\left(Q_{T}\right)}^{2},
$$

if $\lambda \in(0,1 / 2)$, and

$$
\|u\|_{H^{l, l / 2}\left(O_{T}\right)}^{2}=\|u\|_{\widehat{W}_{2}^{l, l / 2}\left(O_{T}\right)}^{2}+\sum_{0 \leqslant j<(l-1) / 2} \sup _{t<T}\left\|D_{t}^{j} u(\cdot, t)\right\|_{W_{2}^{l-1-2 j}(\Omega)}^{2} .
$$

Similar norms can be introduced on the manifold $G_{T}=\mathcal{G} \times(0, T)$. The advantages furnished by working with $H$-norms are discussed in [22, Propositions 1.1 and 1.2].

We prove the following theorem.

THEOREM 1 Let $\boldsymbol{u}_{0} \in W_{2}^{l+1}\left(\mathfrak{F}_{1}\right), \rho_{0} \in W_{2}^{l+2}(\mathcal{G}), \boldsymbol{h}_{0} \in W_{2}^{l^{\prime}+1}\left(\mathfrak{F}_{i}\right), i=1,2,3$, with $1 / 2<l^{\prime}<$ $l<1$, and let the compatibility conditions

$$
\begin{aligned}
& \widetilde{\nabla} \cdot \boldsymbol{u}_{0}=0, \quad y \in \mathcal{F}_{1}, \quad \widetilde{S}\left(\boldsymbol{u}_{0}\right) \boldsymbol{n}_{0}\left(e_{\rho_{0}}\right)-\boldsymbol{n}_{0}\left(\boldsymbol{n}_{0} \cdot \widetilde{S}\left(\boldsymbol{u}_{0}\right) \boldsymbol{n}_{0}\right)=0, \quad y \in \mathcal{G}, \\
& \nabla \cdot \boldsymbol{h}_{0}=0, \quad y \in \mathcal{F}_{i}, \quad i=1,2,3, \\
& \operatorname{rot} \mathbb{P}\left(y, \rho_{0}\right) \boldsymbol{h}_{0}=0, \quad y \in \mathcal{F}_{2}, \\
& {\left[\mu \boldsymbol{h}_{0} \cdot \boldsymbol{N}\right]=0, \quad\left[\boldsymbol{h}_{0 \tau}\right]=\left(\frac{\widehat{\mathcal{L}}\left(y, \rho_{0}\right) \widehat{\mathcal{L}}^{T} \boldsymbol{N}}{\left|\widehat{\mathcal{L}}^{T} N\right|^{2}}-\boldsymbol{N}\right)\left[\boldsymbol{h}_{0} \cdot \boldsymbol{N}\right], \quad y \in \mathcal{G},} \\
& {\left[\mu \boldsymbol{h}_{0} \cdot \boldsymbol{n}\right]=0, \quad\left[\boldsymbol{h}_{0 \tau}\right]=0, \quad y \in S_{3},} \\
& \left.\boldsymbol{h}_{0} \cdot \boldsymbol{n}\right|_{S}=0 \text {, }
\end{aligned}
$$

and the smallness condition

$$
\left\|\rho_{0}\right\|_{W_{2}^{l+3 / 2}(9)} \leqslant \epsilon \ll 1
$$

be satisfied. Assume also that

$$
\begin{aligned}
& \boldsymbol{f} \in W_{2}^{l / 2}\left(0, T ; W_{2}^{2}\left(\mathbb{R}^{3}\right)\right) \cap W_{2}^{l+2,0}\left(\mathbb{R}^{3} \times\left(0, T_{0}\right)\right), \\
& \boldsymbol{j} \in W_{2}^{l^{\prime} / 2}\left(0, T ; W_{2}^{1}\left(\mathcal{F}_{3}\right)\right) \cap W_{2}^{l^{\prime}+1,0}\left(\mathcal{F}_{3} \times\left(0, T_{0}\right)\right)
\end{aligned}
$$


and that the condition (1.1) holds. Then the problem (2.7)-(2.11) has a unique solution defined in a certain (small) time interval $(0, T)$ with the following regularity properties:

$$
\begin{aligned}
& \boldsymbol{u} \in W_{2}^{2+l .1+l / 2}\left(Q_{T}^{1}\right), \quad \nabla q \in W_{2}^{l, l / 2}\left(Q_{T}^{1}\right), \\
& q \in W_{2}^{l+1 / 2,0}\left(G_{T}\right) \cap W_{2}^{l / 2}\left(0, T ; W_{2}^{1 / 2}(\mathcal{G})\right), \\
& \rho \in W_{2}^{l+5 / 2,0}\left(G_{T}\right) \cap W_{2}^{l / 2}\left(0, T ; W_{2}^{5 / 2}(\mathcal{G})\right), \\
& \rho_{t} \in W_{2}^{l+3 / 2, l / 2+3 / 4}\left(G_{T}\right), \quad \boldsymbol{h}^{(i)} \in W_{2}^{l^{\prime}+2, l^{\prime} / 2+1}\left(Q_{T}^{i}\right), \\
& \boldsymbol{e}^{(i)} \in W_{2}^{l^{\prime}+1,0}\left(Q_{T}^{i}\right) \cap W_{2}^{l^{\prime} / 2}\left(0, T ; W_{2}^{1}\left(\mathfrak{F}_{i}\right)\right),
\end{aligned}
$$

where $Q_{T}^{i}=\mathcal{F}_{i} \times(0, T), G_{T}=\mathcal{G} \times(0, T), \boldsymbol{h}^{(i)}=\left.\boldsymbol{h}\right|_{Q_{T}^{i}}, \boldsymbol{e}^{(i)}=\left.\boldsymbol{e}\right|_{Q_{T}^{i}}, i=1,2,3$. The solution satisfies the inequality

$$
\begin{aligned}
& \|\boldsymbol{u}\|_{H^{l+2, l / 2+1}\left(Q_{T}^{1}\right)}+\|\nabla q\|_{\widehat{W}_{2}^{l, l / 2}\left(Q_{T}^{1}\right)}+\|q\|_{W_{2}^{l+1 / 2,0}\left(G_{T}\right)}+\|q\|_{\widehat{W}_{2}^{l / 2}\left(0, T ; W_{2}^{1 / 2}\left(\mathcal{G}_{T}\right)\right)} \\
& \quad+\|\rho\|_{W_{2}^{l+5 / 2,0}\left(G_{T}\right)}+\|\rho\|_{\widehat{W}_{2}^{l / 2}\left(0, T ; W_{2}^{5 / 2}\left(\mathcal{G}_{T}\right)\right)}+\left\|\rho_{t}\right\|_{\left.H^{l+3 / 2, l / 2+3 / 4}\left(G_{T}\right)\right)} \\
& \quad+\sup _{t<T}\|\rho(\cdot, t)\|_{W_{2}^{l+2}(\mathrm{G})}+\sum_{i=1}^{3}\left\|\boldsymbol{h}^{(i)}\right\|_{H^{l^{\prime}+2, l^{\prime} / 2+1}\left(Q_{T}^{i}\right)} \\
& \quad+\sum_{i=1}^{3}\left(\left\|\boldsymbol{e}^{(i)}\right\|_{W_{2}^{l^{\prime}+1,0}\left(Q_{T}^{i}\right)}+\left\|\boldsymbol{e}^{(i)}\right\|_{\widehat{W}_{2}^{l^{\prime} / 2}\left(0, T ; W_{2}^{1}(\mathrm{G})\right)}\right) \\
& \leqslant c\left(\|\boldsymbol{f}\|_{\widehat{W}_{2}^{l, l / 2}\left(Q_{T}^{1}\right)}+\|\mathcal{H}\|_{W_{2}^{l+1 / 2}(\mathcal{G})}+\left\|\boldsymbol{u}_{0}\right\|_{W_{2}^{l+1}\left(\mathcal{F}_{1}\right)}+\left\|\rho_{0}\right\|_{W_{2}^{l+2}(\mathrm{~g})}\right. \\
& \left.\quad+\sum_{i=1}^{3}\left\|\boldsymbol{h}_{0}\right\|_{W_{2}^{l^{\prime}+1}\left(\Omega_{i 0}\right)}+\|\boldsymbol{j}\|_{W_{2}^{l^{\prime}+1,0}\left(Q_{T}^{3}\right)}+\|\boldsymbol{j}\|_{\widehat{W}_{2}^{l^{\prime} / 2}\left(0, T ; W_{2}^{1}\left(\mathcal{F}_{3}\right)\right)}\right) .
\end{aligned}
$$

For the problem (1.2)-(1.5),(1.7), (1.12), (1.13) this means that it is solvable in the time interval $(0, T)$ and

$$
\begin{aligned}
& \boldsymbol{v} \circ e_{\rho} \in W_{2}^{2+l .1+l / 2}\left(Q_{T}^{1}\right), \quad \nabla p \circ e_{\rho} \in W_{2}^{l, l / 2}\left(Q_{T}^{1}\right), \\
& p \circ e_{\rho} \in W_{2}^{l+1 / 2,0}\left(G_{T}\right) \cap W_{2}^{l / 2}\left(0, T ; W_{2}^{1 / 2}(\mathcal{G})\right), \\
& \rho \in W_{2}^{l+5 / 2,0}\left(G_{T}\right) \cap W_{2}^{l / 2}\left(0, T ; W_{2}^{5 / 2}(\mathcal{G})\right), \\
& \rho_{t} \in W_{2}^{l+3 / 2, l / 2+3 / 4}\left(G_{T}\right), \quad \boldsymbol{H}^{(i)} \circ e_{\rho} \in W_{2}^{l^{\prime}+2, l^{\prime} / 2+1}\left(Q_{T}^{i}\right), \\
& \boldsymbol{E}^{(i)} \circ e_{\rho} \in W_{2}^{l^{\prime}+1,0}\left(Q_{T}^{i}\right) \cap W_{2}^{l^{\prime} / 2}\left(0, T ; W_{2}^{1}\left(\mathcal{F}_{i}\right)\right), \quad i=1,2,3 .
\end{aligned}
$$

Once the solution with the above-mentioned properties is obtained, it can be shown that

$$
\begin{aligned}
& \boldsymbol{h}^{(i)} \in W_{2}^{l+2, l / 2+1}\left(Q_{T}^{i}\right), \\
& \boldsymbol{e}^{(i)} \in W_{2}^{l+1,0}\left(Q_{T}^{i}\right) \cap W_{2}^{l / 2}\left(0, T ; W_{2}^{1}\left(\mathcal{F}_{i}\right)\right), \quad i=1,2,3,
\end{aligned}
$$

provided $\boldsymbol{h}_{0} \in W_{2}^{l+1}\left(\Omega_{i 0}\right), i=1,2,3, \boldsymbol{j} \in W_{2}^{l+1,0}\left(Q_{T}^{3}\right) \cap W_{2}^{l / 2}\left(0, T ; W_{2}^{1}\left(\mathfrak{F}_{3}\right)\right)$ (see Sec. 4).

The restriction $l>1 / 2$ comes from the estimates of nonlinear terms, and $l<1$ can be relaxed and replaced with $l<5 / 2$. For $l>5 / 2$ additional compatibility conditions are required. 
Theorem 1 is proved in the paper [20] under the following extra assumptions: $\boldsymbol{f}=0, \boldsymbol{j}=0$, $\Omega_{3}=\emptyset, \Omega_{1}$ and $\Omega$ are simply connected. In this case the equations $\operatorname{rot} \boldsymbol{H}=0, \quad \nabla \cdot \boldsymbol{H}=0, \quad x \in$ $\Omega_{2 t}$ imply

$$
\boldsymbol{H}^{(2)}=\nabla \varphi
$$

where $\varphi$ is a solution of the Neumann problem

$$
\nabla^{2} \varphi(x, t)=0, \quad x \in \Omega_{2 t}, \quad \mu_{2} \frac{\partial \varphi}{\partial n}=\mu_{1} \boldsymbol{H}^{(1)} \cdot \boldsymbol{n}, \quad x \in \Gamma_{t}, \quad \frac{\partial \varphi}{\partial n}=0, \quad x \in S
$$

and $\boldsymbol{H}^{(i)}(x, t)=\left.\boldsymbol{H}(x, t)\right|_{x \in \Omega_{i t}}$. Hence $\boldsymbol{H}^{(2)}$ is completely determined by $\left.\boldsymbol{H}^{(1)} \cdot \boldsymbol{n}\right|_{x \in \Gamma_{t}}$, which permits to exclude $\boldsymbol{E}$ and to work only with the function $\boldsymbol{H}^{(1)}$ satisfying the relations

$$
\left\{\begin{array}{l}
\mu_{1} \boldsymbol{H}_{t}+\alpha^{-1} \text { rotrot } \boldsymbol{H}-\mu_{1} \operatorname{rot}(\boldsymbol{v} \times \boldsymbol{H})=0, \\
\nabla \cdot \boldsymbol{H}(x, t)=0, \quad x \in \Omega_{1 t}, \\
\boldsymbol{H}_{\tau}=\mathbb{B}\left(\boldsymbol{H}^{(1)} \cdot \boldsymbol{n}\right), \quad x \in \Gamma_{t} \\
\boldsymbol{H}(x, 0)=\boldsymbol{H}_{0}(x), \quad x \in \Omega_{10}
\end{array}\right.
$$

where $\mathbb{B}$ is a non-local linear operator defined through (2.21). In the general case (2.20) is not true, nevertheless, one can separate the determination of $\boldsymbol{u}, \rho, \boldsymbol{h}$ from that of $\boldsymbol{e}$. Let $\boldsymbol{\psi} \in W_{2}^{1,0}\left(Q_{T}^{i}\right)$, $i=1,2,3$ be a test vector field satisfying the conditions

$$
\begin{aligned}
\nabla \cdot \boldsymbol{\psi}(y, t)= & 0, \quad y \in \mathcal{F}_{1} \cup \mathcal{F}_{2} \cup \mathcal{F}_{3}, \quad \operatorname{rot} \boldsymbol{\psi}(y, t)=0, \quad y \in \mathcal{F}_{2}, \\
& {[\mu \boldsymbol{\psi} \cdot \boldsymbol{N}]=0, \quad\left[\boldsymbol{\psi}_{\tau}\right]=0, \quad y \in \mathcal{G}, } \\
& {[\mu \boldsymbol{\psi} \cdot \boldsymbol{n}]=0, \quad\left[\boldsymbol{\psi}_{\tau}\right]=0, \quad y \in S_{3}, } \\
& \boldsymbol{\psi} \cdot \boldsymbol{n}=0, \quad y \in S .
\end{aligned}
$$

Since, in view of (2.13),

$$
\int_{\Omega} \operatorname{rot} \boldsymbol{P} \boldsymbol{e}(y, t) \cdot \boldsymbol{\psi}(y, t) d y=\int_{\Omega} \boldsymbol{P} \boldsymbol{e} \cdot \operatorname{rot} \boldsymbol{\psi} d y+\int_{\mathrm{g}} \frac{\boldsymbol{u} \cdot \widehat{\mathcal{L}}^{T} \boldsymbol{N}}{J}[\mu \boldsymbol{h}] \cdot \boldsymbol{\psi} d S,
$$

it is easy to obtain from the equations (2.8) the integral identity

$$
\begin{array}{r}
\int_{0}^{T} \int_{\Omega} \mu\left(\boldsymbol{h}_{t}-\boldsymbol{\Phi}(\boldsymbol{h}, \rho)\right) \cdot \boldsymbol{\psi}(y, t) d y d t+\int_{0}^{T} \int_{\mathcal{F}_{1} \cup \mathcal{F}_{3}} \alpha^{-1} \operatorname{ProtP} \boldsymbol{h} \cdot \operatorname{rot} \boldsymbol{\psi}(y, t) d y d t \\
-\mu_{1} \int_{0}^{T} \int_{\mathcal{F}_{1}}\left(\mathcal{L}^{-1} \boldsymbol{u} \times \boldsymbol{h}\right) \cdot \operatorname{rot} \boldsymbol{\psi}(y, t) d y d t+\int_{0}^{T} d t \int_{\mathcal{S}} \boldsymbol{\Psi}(\boldsymbol{u}, \boldsymbol{h}, \rho) \cdot \boldsymbol{\psi} d S \\
=\int_{0}^{T} \int_{\mathcal{F}_{3}} \alpha^{-1} \boldsymbol{j}(y, t) \cdot \operatorname{rot} \boldsymbol{\psi}(y, t) d y d t,
\end{array}
$$

where

$$
\begin{aligned}
& \boldsymbol{\Phi}(\boldsymbol{h}, \rho)=\frac{1}{J} \widehat{\mathcal{L}}_{t} \mathcal{L} \boldsymbol{h}+\rho_{t}^{*} \widehat{\mathcal{L}}\left(\mathcal{L}^{-1} \boldsymbol{N}^{*}(y) \cdot \nabla\right) \frac{1}{J} \mathcal{L} \boldsymbol{h}, \\
& \boldsymbol{\Psi}(\boldsymbol{u}, \boldsymbol{h}, \rho)=\frac{\boldsymbol{u} \cdot \widehat{\mathcal{L}}^{T} \boldsymbol{N}}{\boldsymbol{J}}[\mu \boldsymbol{h}] .
\end{aligned}
$$

This identity, together with the initial and boundary conditions for $\boldsymbol{h}$ and the equation $\operatorname{rot} \boldsymbol{P} \boldsymbol{h}=0$ in $\Omega_{2}$, constitutes a nonlinear problem studied in Sec. 4 . 


\section{Linear problems}

The proof of the solvability of the problem (2.7)-(2.10) is based on the analysis of the following non-homogeneous linear problems:

1. Find $(\boldsymbol{v}, p, \rho)$ such that

$$
\left\{\begin{array}{l}
\boldsymbol{v}_{t}-v \nabla^{2} \boldsymbol{v}+\boldsymbol{m}(y, t) \rho^{*}+\nabla p=\boldsymbol{f}(y, t) \\
\nabla \cdot \boldsymbol{v}=f(y, t)=\nabla \cdot \boldsymbol{F}(y, t), \quad y \in \mathcal{F}_{1}, \quad t>0 \\
T(\boldsymbol{v}, p) \boldsymbol{N}(y)+\sigma \boldsymbol{N}(y) \mathfrak{B} \rho=\boldsymbol{d}(y, t), \\
\rho_{t}+\boldsymbol{V}(x) \cdot \nabla_{\tau} \rho-\boldsymbol{v} \cdot \boldsymbol{N}(y)=g(y, t), \quad y \in \mathcal{G}, \\
\boldsymbol{v}(y, 0)=\boldsymbol{v}_{0}(y), \quad y \in \mathcal{F}_{1}, \quad \rho(y, 0)=\rho_{0}(y), \quad y \in \mathcal{G}
\end{array}\right.
$$

where $\mathfrak{B} \rho=-\Delta_{\mathrm{g}} \rho-b(y) \rho, b=\left(\mathcal{H}_{G}^{2}-2 \mathcal{K}_{G}\right), \Delta_{\mathrm{g}}$ is the Laplace-Beltrami operator on $\mathcal{G}$,

2. Find the vector field $\boldsymbol{H}(y, t)$, satisfying the equations

$$
\begin{aligned}
& \operatorname{rot} \boldsymbol{H}(y, t)=\operatorname{rot\ell }(y, t), \quad y \in \mathcal{F}_{2}, \quad \boldsymbol{H}(y, 0)=\boldsymbol{H}_{0}(y), \quad y \in \mathcal{F}_{1} \cup \mathcal{F}_{2} \cup \mathcal{F}_{3}, \\
& {[\mu \boldsymbol{H} \cdot \boldsymbol{N}]=0, \quad\left[\boldsymbol{H}_{\tau}\right]=\boldsymbol{a}, \quad y \in \mathcal{G}, \quad[\mu \boldsymbol{H} \cdot \boldsymbol{n}]=0, \quad\left[\boldsymbol{H}_{\tau}\right]=0, \quad y \in S_{3},} \\
& \boldsymbol{H} \cdot \boldsymbol{n}=0, \quad y \in S .
\end{aligned}
$$

and the integral identity

$$
\begin{aligned}
& \int_{0}^{T} \int_{\Omega} \mu \boldsymbol{H}_{t} \cdot \boldsymbol{\psi}(y, t) d y d t+\int_{0}^{T} \int_{\mathcal{F}_{1} \cup \mathcal{F}_{3}} \alpha^{-1} \operatorname{rot} \boldsymbol{H} \cdot \operatorname{rot} \psi(y, t) d y d t \\
& =\int_{0}^{T} \int_{\mathcal{F}_{1} \cup \mathcal{F}_{3}} \boldsymbol{G}_{1}(y, t) \cdot \operatorname{rot} \psi(y, t) d y d t+\int_{0}^{T} \int_{\Omega} \mu \boldsymbol{G}_{2}(y, t) \cdot \psi(y, t) d y d t,
\end{aligned}
$$

with the same kind of the test function $\psi(y, t)$ as in (2.24).

In addition, we need to consider the auxiliary problem

$$
\left\{\begin{array}{l}
\operatorname{roth}(y)=\boldsymbol{k}(y), \quad \nabla \cdot \boldsymbol{h}=0, \quad y \in \mathcal{F}_{1} \cup \mathcal{F}_{2} \cup \mathcal{F}_{3}, \\
{[\mu \boldsymbol{h} \cdot \boldsymbol{N}]=0, \quad\left[\boldsymbol{h}_{\tau}\right]=\boldsymbol{a}, \quad y \in \mathcal{G}} \\
{[\mu \boldsymbol{h} \cdot \boldsymbol{n}]=0, \quad\left[\boldsymbol{h}_{\tau}\right]=0, \quad y \in S_{3}} \\
\boldsymbol{h} \cdot \boldsymbol{n}(y)=0, \quad y \in S
\end{array}\right.
$$

We start with the existence theorem for the problem (3.1).

TheOREM 2 Assume that $l \in(1 / 2,1)$ and that the data of the problem (3.1) possess the following regularity properties: $f \in W_{2}^{l, l / 2}\left(Q_{T}^{1}\right), f \in W_{2}^{l+1,0}\left(Q_{T}^{1}\right), \boldsymbol{F} \in W_{2}^{0,1+l / 2}\left(Q_{T}^{1}\right)$, $\boldsymbol{d} \cdot \boldsymbol{N} \in W_{2}^{l+1 / 2,0}\left(G_{T}\right) \cap W_{2}^{l / 2}\left(0, T ; W_{2}^{1 / 2}(\mathcal{G})\right), \boldsymbol{d}-\boldsymbol{N}(\boldsymbol{d} \cdot \boldsymbol{N}) \in W_{2}^{l+1 / 2, l / 2+1 / 4}\left(G_{T}\right), g \in$ $W_{2}^{l+3 / 2, l / 2+3 / 4}\left(G_{T}\right), v_{0} \in W_{2}^{l+1}\left(\mathcal{F}_{1}\right), \rho_{0} \in W_{2}^{l+2}(\mathcal{G})$, where $T<\infty, Q_{T}^{1}=\mathcal{F}_{1} \times(0, T)$, $G_{T}=\mathcal{G} \times(0, T)$. Moreover, let $\boldsymbol{m} \in W_{2}^{l, l / 2}\left(Q_{T}^{1}\right), \boldsymbol{V} \in W_{2}^{l+3 / 2}(\mathcal{G})$ and let the compatibility conditions

$$
\begin{aligned}
& \nabla \cdot \boldsymbol{v}_{0}(x)=f(x, 0), \quad x \in \mathcal{F}_{1}, \\
& v \Pi_{\mathrm{g}} S\left(\boldsymbol{v}_{0}\right) \boldsymbol{N}=\Pi_{\mathrm{g}} \boldsymbol{d}(x, 0), \quad x \in \mathcal{G},
\end{aligned}
$$


be satisfied, where

$$
\Pi_{\mathrm{g}} \boldsymbol{d}=\boldsymbol{d}-\boldsymbol{N}(\boldsymbol{N} \cdot \boldsymbol{d})
$$

is the tangential component of $\boldsymbol{d}$ on $\mathcal{G}$. Then the problem (3.1) has a unique solution $\boldsymbol{v}, p, \rho$ such that

$$
\begin{array}{ll}
v \in W_{2}^{l+2, l / 2+1}\left(Q_{T}^{1}\right), \quad \nabla p \in W_{2}^{l, l / 2}\left(Q_{T}^{1}\right), \quad p \in W_{2}^{l+1 / 2,0}\left(G_{T}\right) \cap W_{2}^{l / 2}\left(0, T ; W_{2}^{1 / 2}(\mathcal{G})\right), \\
\rho \in W_{2}^{l+5 / 2,0}\left(G_{T}\right) \cap W_{2}^{l / 2}\left(0, T ; W_{2}^{5 / 2}(\mathcal{G})\right), \quad \rho_{t} \in W_{2}^{l+3 / 2, l / 2+3 / 4}\left(G_{T}\right),
\end{array}
$$

and the solution satisfies the inequality

$$
\begin{aligned}
& \|\boldsymbol{v}\|_{H^{l+2, l / 2+1}\left(Q_{T}^{1}\right)}+\|\nabla p\|_{\widehat{W}_{2}^{l, l / 2}\left(Q_{T}^{1}\right)}+\|p\|_{W_{2}^{l+1 / 2,0}\left(G_{T}\right)}+\|p\|_{\widehat{W}_{2}^{l / 2}\left(0, T ; W_{2}^{1 / 2}\left(\mathcal{G}_{T}\right)\right)} \\
& \quad+\|\rho\|_{W_{2}^{l+5 / 2,0}\left(G_{T}\right)}+\|\rho\|_{\widehat{W}_{2}^{l / 2}\left(0, T ; W_{2}^{5 / 2}\left(G_{T}\right)\right)}+\left\|\rho_{t}\right\|_{H^{l+3 / 2, l / 2+3 / 4}\left(G_{T}\right)} \\
& \leqslant c\left(\|\boldsymbol{f}\|_{\widehat{W}_{2}^{l, l / 2}\left(Q_{T}^{1}\right)}+\|f\|_{W_{2}^{l+1,0}\left(Q_{T}^{1}\right)}+\|\boldsymbol{F}\|_{\widehat{W}_{2}^{0,1+l / 2}\left(Q_{T}^{1}\right)}\right. \\
& \quad+\left\|\Pi_{\mathrm{g}} \boldsymbol{d}\right\|_{H^{l+1 / 2, l / 2+1 / 4}\left(G_{T}\right)}+\|\boldsymbol{d} \cdot \boldsymbol{N}\|_{W_{2}^{l+1 / 2,0}\left(G_{T}\right)}+\|\boldsymbol{d} \cdot \boldsymbol{N}\|_{\widehat{W}_{2}^{l / 2}\left(0, T ; W_{2}^{1 / 2}(\mathrm{~g})\right)} \\
& \left.\quad+\|g\|_{H^{l+3 / 2, l / 2+3 / 4}\left(G_{T}\right)}+\left\|\boldsymbol{u}_{0}\right\|_{W_{2}^{l+1}\left(\mathcal{F}_{1}\right)}+\left\|\rho_{0}\right\|_{W_{2}^{l+2}(\mathrm{G})}\right) .
\end{aligned}
$$

This theorem is proved in [23] in the case $\boldsymbol{m}=0$. The term $\boldsymbol{m} \rho^{*}$ in (3.1) is weak and can be estimated by the interpolation inequality

$$
\left\|m \rho^{*}\right\|_{\widehat{W}_{2}^{l, l / 2}\left(Q_{T}^{1}\right)} \leqslant c \eta\left(\|\rho\|_{W_{2}^{l+5 / 2,0}\left(G_{T}\right)}+\left\|\rho_{t}\right\|_{\left.W_{2}^{l+3 / 2, l / 2+3 / 4}\left(G_{T}\right)\right)}\right)+c(\eta)\|\rho\|_{L_{2}\left(G_{T}\right)},
$$

with arbitrarily small $\eta$, so the result of [23] extends to the case $\boldsymbol{m} \neq 0$ in a standard way.

Before discussing the problems (3.2), (3.3) and (3.4), we recall some basic results concerning the space $L_{2}(D)$ of square summable vector fields given in the domain $\Phi \subset \mathbb{R}^{3}$ with a smooth connected boundary $\partial Ф$. We introduce the finite dimensional spaces of the Neumann and Dirichlet vector fields

$$
\begin{array}{llll}
U_{n}(\Phi)=\left\{\boldsymbol{u} \in W_{2}^{1}(\Phi):\right. & \nabla \cdot \boldsymbol{u}=0, & \operatorname{rot} \boldsymbol{u}=0, & \left.\left.\boldsymbol{u} \cdot \boldsymbol{n}\right|_{\partial \Phi}=0\right\}, \\
U_{d}(\Phi)=\left\{\boldsymbol{v} \in W_{2}^{1}(\Phi):\right. & \nabla \cdot \boldsymbol{v}=0, & \operatorname{rot} \boldsymbol{v}=0, & \left.\left.\boldsymbol{v}_{\tau}\right|_{\partial \Phi}=0\right\} .
\end{array}
$$

The dimensions of these spaces are equal to the first and the second Betti numbers of $\Phi, b_{1}(\Phi)$ and $b_{2}(D)$, respectively. Following [24, 17], we recall the structure of the Neumann vector fields. In the case $b_{1}(\Phi)>0$ there exist $b_{1}(\Phi)$ closed contours $\mathcal{C} \in \mathbb{R}^{3} \backslash \bar{\Phi}$ generating the first homology group of $\mathbb{R}^{3} \backslash \bar{D}$, end every such contour generates the Neumann vector field of the form

$$
\boldsymbol{u}=\boldsymbol{u}_{1}+\boldsymbol{u}_{2}
$$

with $\boldsymbol{u}_{1}$ given through the Biot-Savart law

$$
\boldsymbol{u}_{1}(x)=\int_{\mathfrak{e}} \frac{x-y}{|x-y|^{3}} \times d \boldsymbol{l}_{y}
$$

and $\boldsymbol{u}_{2}=\nabla \varphi$

$$
\triangle \varphi=0, \quad \text { in } D,\left.\quad \frac{\partial \varphi}{\partial n}\right|_{\partial D}=-\left.\boldsymbol{u}_{1} \cdot \boldsymbol{n}\right|_{\partial D} .
$$


As for $b_{2}(\Phi)$, it is equal to the number of connected components $\Gamma_{j}$ of the boundary of $\Phi$ minus one (denoted by $\Gamma_{0}$ ), and the basis in $U_{d}(D)$ consists of vector fields $v_{k}(x)=\nabla \Phi_{k}(x)$, where $\Phi_{k}$ is a solution of the Dirichlet problem

$$
\Delta^{2} \Phi_{k}=0,\left.\quad \Phi_{k}\right|_{\Gamma_{j}}=\delta_{k j}, \quad k, j=1, \ldots, b_{2}(\Phi),\left.\quad \Phi_{k}\right|_{\Gamma_{0}}=0 .
$$

We also recall the Weyl decomposition formula for the space $L_{2}(D)$ [25]:

$$
L_{2}(D)=\stackrel{\circ}{G}(D) \oplus U(D) \oplus \stackrel{\circ}{J}(\Phi)=G(D) \oplus \stackrel{\circ}{J}(\Phi)=\stackrel{\circ}{G}(\Phi) \oplus J(D),
$$

where

$$
\begin{aligned}
& \stackrel{\circ}{G}(\Phi)=\left\{\boldsymbol{u} \in L_{2}(\Phi): \quad \boldsymbol{u}=\nabla \varphi, \quad \varphi \in W_{2}^{1}(Ф),\left.\quad \varphi\right|_{\partial Ф}=0\right\}, \\
& U(D)=\left\{\boldsymbol{u} \in L_{2}(\Phi): \quad \boldsymbol{u}=\nabla \psi, \quad \psi \in W_{2}^{1}(\Phi), \quad \nabla^{2} \psi=0\right\} \text {, } \\
& \stackrel{\circ}{J}(\Phi)=\left\{\boldsymbol{u} \in L_{2}(\Phi): \quad \nabla \cdot \boldsymbol{u}=0,\left.\quad \boldsymbol{u} \cdot \boldsymbol{n}\right|_{\partial \Phi}=0\right\} ;
\end{aligned}
$$

$G(\Phi)=\stackrel{\circ}{G}(\Phi) \oplus U(D)$ is the space of the potential vector fields and $J(D)=\stackrel{\circ}{J}(\Phi) \oplus U(D)$ is the space of divergence free vector fields.

It is clear that $U_{n}(\Phi) \subset \stackrel{\circ}{J}(\Phi)$ and $U_{d}(\Phi) \subset U(D)$.

Following [17], we introduce in the domain $\Omega=\overline{\mathfrak{F}}_{1} \cup \mathcal{F}_{2} \cup \overline{\mathfrak{F}}_{3}$ the $b_{1}(\Omega)$-dimensional space of the modified Neumann vector fields $\widetilde{U}_{n}(\Omega)$ whose elements $\widetilde{\boldsymbol{u}}_{k}(y)$ satisfy the relations

$$
\begin{aligned}
& \operatorname{rot} \widetilde{\boldsymbol{u}}_{q}(x)=0, \quad \nabla \cdot \widetilde{\boldsymbol{u}}_{q}(x)=0, \quad x \in \mathcal{F}_{i}, \quad i=1,2,3, \\
& {\left[\mu \widetilde{\boldsymbol{u}}_{q} \cdot \boldsymbol{N}\right]=0, \quad\left[\widetilde{\boldsymbol{u}}_{q \tau}\right]=0, \quad x \in \mathcal{G},} \\
& {\left[\mu \widetilde{\boldsymbol{u}}_{q} \cdot \boldsymbol{n}\right]=0, \quad\left[\widetilde{\boldsymbol{u}}_{q \tau}\right]=0, \quad x \in S_{3},} \\
& \widetilde{\boldsymbol{u}}_{q}(x) \cdot \boldsymbol{n}(x)=0, \quad x \in S .
\end{aligned}
$$

It is clear that $\widetilde{\boldsymbol{u}}_{q}(x)=\boldsymbol{u}_{q}(x)+\nabla \omega_{q}(x): \boldsymbol{u}_{q} \in U_{n}(\Omega)$,

$$
\left\{\begin{array}{l}
\nabla^{2} \omega_{q}(x)=0, \quad x \in \mathcal{F}_{i}, \quad i=1,2,3 \\
{\left[\mu \frac{\partial \omega_{q}}{\partial N}\right]=-[\mu] \boldsymbol{u}_{q} \cdot \boldsymbol{N}, \quad\left[\omega_{q}\right]=0, \quad x \in \mathcal{G}} \\
{\left[\mu \frac{\partial \omega_{q}}{\partial n}\right]=-[\mu] \boldsymbol{u}_{q} \cdot \boldsymbol{n}, \quad\left[\omega_{q}\right]=0, \quad x \in S_{3}} \\
\frac{\partial \omega_{q}}{\partial n}=0, \quad x \in S .
\end{array}\right.
$$

We denote by $\mathcal{H}^{l}(\Omega)$ the space of the vector fields $\psi \in W_{2}^{l}\left(\mathfrak{F}_{i}\right), i=1,2,3$, satisfying (2.22), (2.23). For $l<1 / 2$ the boundary conditions (2.23) are replaced with

$$
[\mu \boldsymbol{\psi} \cdot \boldsymbol{N}]=0, \quad y \in \mathcal{G}, \quad[\mu \boldsymbol{\psi} \cdot \boldsymbol{n}]=0, \quad y \in S_{3}, \quad \boldsymbol{\psi} \cdot \boldsymbol{n}=0, \quad y \in S,
$$

because $\boldsymbol{\psi}_{\tau}$ has no sense in this case. By $\mathcal{H}^{0}(\Omega)$ we mean the closure of $\mathcal{H}^{l}(\Omega)$ in $L_{2}(\Omega)$. If $\boldsymbol{\psi} \in$ $\mathcal{H}^{0}(\Omega)$, then $\boldsymbol{\psi}^{(i)} \in J\left(\mathcal{F}_{i}\right), i=1.3$. and $\boldsymbol{\psi}^{(2)}=\nabla \varphi(y)+\sum_{i=1}^{b_{1}\left(\mathcal{F}_{2}\right)} c_{j} \boldsymbol{w}_{j}(y)$, where $\boldsymbol{w}_{j} \in U_{n}\left(\mathcal{F}_{2}\right)$ 
and $\varphi$ is the solution of the Neumann problem

$$
\left\{\begin{array}{l}
\nabla^{2} \varphi(y)=0, \quad y \in \mathcal{F}_{2}, \quad \mu_{2} \frac{\partial \varphi}{\partial N}=\mu_{1} \boldsymbol{\psi}^{(1)} \cdot \boldsymbol{N}, \quad y \in \mathcal{G}, \\
\mu_{2} \frac{\partial \varphi}{\partial n}=\mu_{3} \boldsymbol{\psi}^{(3)} \cdot \boldsymbol{n}, \quad y \in \mathcal{S}_{3}, \quad \frac{\partial \varphi}{\partial n}=0 \quad y \in S .
\end{array}\right.
$$

Along with (3.7), we shall use in $\Omega$ the decomposition

$$
L_{2, \mu}(\Omega)=G_{\mu}(\Omega) \oplus \stackrel{\circ}{J}_{\mu}(\Omega)=\stackrel{\circ}{G}_{\mu}(\Omega) \oplus J_{\mu}(\Omega),
$$

where $L_{2, \mu}(\Omega)$ is the space $L_{2}(\Omega)$ supplied with the scalar product

$$
(\boldsymbol{u}, \boldsymbol{v})_{\mu}=\int_{\Omega} \mu \boldsymbol{u} \cdot \boldsymbol{v} d y
$$

$\stackrel{\circ}{J}_{\mu}(\Omega)$ is the space of divergence free vector fields satisfying the conditions (2.23), and $G_{\mu}(\Omega)=$ $G(\Omega)=\left\{\boldsymbol{u}=\nabla \varphi, \varphi \in W_{2}^{1}(\Omega)\right\}$. The projection operator $P_{\mu}$ on $\stackrel{\circ}{J}_{\mu}(\Omega)$ is defined by $P_{\mu} \boldsymbol{u}=$ $\boldsymbol{u}-\nabla \varphi$, where $\varphi \in W_{2}^{1}(\Omega)$ is the solution of the problem

$$
\left\{\begin{array}{l}
\nabla^{2} \varphi=\nabla \cdot \boldsymbol{u}(y),\left.\quad[\varphi]\right|_{\mathrm{G} \cup S_{3}}=0,\left.\quad\left[\mu \frac{\partial \varphi}{\partial N}\right]\right|_{\mathrm{S}}=\left.[\mu \boldsymbol{u} \cdot \boldsymbol{N}]\right|_{\mathrm{g}}, \\
{\left.\left[\mu \frac{\partial \varphi}{\partial n}\right]\right|_{S_{3}}=\left.[\mu \boldsymbol{u} \cdot \boldsymbol{n}]\right|_{S_{3}},\left.\quad \frac{\partial \varphi}{\partial n}\right|_{S}=\left.\boldsymbol{u} \cdot \boldsymbol{n}\right|_{S},}
\end{array}\right.
$$

or, in a weak form,

$$
\int_{\Omega} \mu \nabla \varphi \cdot \nabla \eta d y=\int_{\Omega} \mu \boldsymbol{u} \cdot \nabla \eta d y, \quad \forall \eta \in W_{2}^{1}(\Omega),
$$

and it is continuous not only in $L_{2}(\Omega)$, but also in $\cup_{i=1}^{3} W_{2}^{r}\left(\mathcal{F}_{i}\right)$, which is a consequence of the estimates of the solution of (3.12).

It is clear that $\mathcal{H}^{l}(\Omega), \widetilde{U}_{n}(\Omega) \subset \stackrel{\circ}{J}_{\mu}(\Omega)$. We denote by $P_{\mathcal{H}} \boldsymbol{f}, \boldsymbol{f} \in \stackrel{\circ}{J}_{\mu}(\Omega)$, the projection of $\boldsymbol{f}$ on $\mathcal{H}^{0}$ and by $P_{\mathcal{H}} f$ the projection of $\boldsymbol{f}$ on the subspace $\mathcal{H}_{\perp}^{0} \subset \mathcal{H}^{0}$ whose elements are orthogonal to $\widetilde{U}_{n}(\Omega)$ :

$$
\int_{\Omega} \mu \psi \cdot \widetilde{\boldsymbol{u}}_{q} d y=0, \quad q=1, \ldots, b_{1}(\Omega) .
$$

It is easily verified that for $f \in J_{\mu}(\Omega)$

$$
P_{\mathcal{H}} \boldsymbol{f}(y)=\left\{\begin{array}{l}
\boldsymbol{f}^{(i)}(y), \quad y \in \mathcal{F}_{i}, \quad i=1,3, \\
\nabla \varphi(y)+\sum_{j=1}^{b_{1}\left(\mathcal{F}_{2}\right)} a_{j} \boldsymbol{w}_{j}(y), \quad y \in \mathcal{F}_{2},
\end{array}\right.
$$

where $\varphi(y)$ is a solution of the Neumann problem

$$
\left\{\begin{array}{l}
\nabla^{2} \varphi(y)=0, \quad y \in \mathcal{F}_{2}, \\
\left.\frac{\partial \varphi}{\partial n}\right|_{S}=0, \quad \frac{\partial \varphi}{\partial N}=f^{(2)}(y) \cdot N, \quad y \in \mathcal{G} \cup S_{3}
\end{array}\right.
$$


(note that $[\mu \boldsymbol{f}]=0$ on $G \cup S_{3}$ ), $\boldsymbol{w}_{j}(y)$ are the Neumann vector fields in $\mathcal{F}_{2}$ orthonormalized in $L_{2}\left(\mathcal{F}_{2}\right)$ and

$$
a_{k}=\int_{\mathfrak{F}_{2}} \boldsymbol{f}^{(2)}(y) \cdot \boldsymbol{w}_{k}(y) d y, \quad k=1, \ldots, b_{1}\left(\mathfrak{F}_{2}\right) .
$$

As $P_{\mu}$, the operators $P_{\mathcal{H}}$ and $P_{\mathcal{H}_{\perp}}$ are continuous not only in $L_{2}(\Omega)$, but also in $\cup_{i=1}^{3} W_{2}^{r}\left(\mathcal{F}_{i}\right)$.

Now we turn to the problem (3.4).

THEOREM 3 If $\boldsymbol{k} \in W_{2}^{l+1}\left(\mathfrak{F}_{i}\right), i=1,2,3$ and the conditions

$$
\begin{aligned}
& \nabla \cdot \boldsymbol{k}(y, t)=0, \quad y \in \mathcal{F}_{1} \cup \mathcal{F}_{2} \cup \mathcal{F}_{3}, \\
& \left.\boldsymbol{k} \cdot \boldsymbol{n}\right|_{S}=0, \quad[\boldsymbol{k} \cdot \boldsymbol{n}]_{S_{3}}=0, \quad[\boldsymbol{k} \cdot \boldsymbol{N}]=\boldsymbol{N} \cdot \operatorname{rota}, \quad y \in \mathcal{G}
\end{aligned}
$$

hold, then the problem (3.4) has a unique solution $\boldsymbol{h} \in W_{2}^{l+2}\left(\mathcal{F}_{i}\right), i=1,2,3$, orthogonal to the space $\widetilde{U}_{n}$ :

$$
\int_{\Omega} \mu \boldsymbol{h} \cdot \widetilde{\boldsymbol{u}}_{q} d x=0, \quad q=1, \ldots, b_{1}(\Omega) .
$$

The solution satisfies the inequality

$$
\sum_{i=1}^{3}\|\boldsymbol{h}\|_{W_{2}^{l+2}\left(\mathcal{F}_{i}\right)} \leqslant c\left(\sum_{i=1}^{3}\|\boldsymbol{k}\|_{W_{2}^{l+1}\left(\mathcal{F}_{i}\right)}+\|\boldsymbol{a}\|_{W_{2}^{l+3 / 2}(\mathcal{G})}\right) .
$$

Moreover, if

$$
\boldsymbol{k}=\operatorname{rot} \boldsymbol{K},\left.\quad\left[\boldsymbol{K}_{\tau}\right]\right|_{S_{3}}=0,\left.\quad \boldsymbol{K}_{\tau}\right|_{S}=0, \quad\left[\boldsymbol{K}_{\tau}\right]-\left.\boldsymbol{\alpha}\right|_{\oint}=0
$$

and $\boldsymbol{a}$ is representable in the form $\boldsymbol{a}=[\boldsymbol{A}]_{G}$ with $\boldsymbol{A}^{(3)}=0, \boldsymbol{A}=0$ near $S$ and $S_{3},\left.\boldsymbol{A}^{(1)} \cdot \boldsymbol{N}\right|_{\mathrm{g}}=$ $\left.\boldsymbol{A}^{(2)} \cdot N\right|_{\mathrm{g}}=0$, then

$$
\|\boldsymbol{h}\|_{L_{2}(\Omega)} \leqslant c\left(\|\boldsymbol{K}\|_{L_{2}(\Omega)}+\|\boldsymbol{A}\|_{L_{2}(\Omega)}\right) .
$$

Proof. Following [20], we construct the solution in the form

$$
\boldsymbol{h}(y)=\boldsymbol{a}^{*}+\nabla \boldsymbol{\psi}+\boldsymbol{\xi}+\sum_{j=1}^{b_{1}(\Omega)} c_{j} \widetilde{\boldsymbol{u}}_{j}
$$

where $\boldsymbol{a}^{*}$ is the extension of $\boldsymbol{a}$ into $\Omega$ such that $\boldsymbol{a}^{*}(y)=0$ for $y \in \mathcal{F}_{2} \cup \mathcal{F}_{3}$ and

$$
\left\|\boldsymbol{a}^{*}\right\|_{W_{2}^{l+2}\left(\mathcal{F}_{1}\right)} \leqslant c\|\boldsymbol{a}\|_{W_{2}^{l+3 / 2}(\mathcal{G})}
$$

The functions $\psi(x)$ and $\xi$ we define as solutions to the problems

$$
\begin{aligned}
& \left\{\begin{array}{l}
\nabla^{2} \psi=-\nabla \cdot \boldsymbol{a}^{*}(x), \quad x \in \mathcal{F}_{1} \cup \mathcal{F}_{2} \cup \mathcal{F}_{3}, \\
{[\psi]=0, \quad\left[\mu \frac{\partial \psi}{\partial N}\right]=0, \quad x \in \mathcal{G}, \quad[\psi]=0, \quad\left[\mu \frac{\partial \psi}{\partial n}\right]=0, \quad x \in S_{3},\left.\quad \frac{\partial \psi}{\partial n}\right|_{S}=0 ;}
\end{array}\right. \\
& \left\{\begin{array}{l}
\operatorname{rot} \boldsymbol{\xi}=\boldsymbol{k}(x)-\operatorname{rota}^{*}(x), \quad \nabla \cdot \boldsymbol{\xi}=0, \quad x \in \mathcal{F}_{1} \cup \mathcal{F}_{2} \cup \mathcal{F}_{3}, \\
{[\mu \boldsymbol{\xi} \cdot \boldsymbol{N}]=0, \quad\left[\xi_{\tau}\right]=0, \quad x \in \mathcal{G}, \quad[\mu \xi \cdot \boldsymbol{n}]=0, \quad\left[\xi_{\tau}\right]=0, \quad x \in S_{3},\left.\quad \boldsymbol{\xi} \cdot \boldsymbol{n}\right|_{S}=0 .}
\end{array}\right.
\end{aligned}
$$


The solution of (3.21) has the form $\boldsymbol{\xi}(x)=\boldsymbol{\xi}_{1}+\nabla \omega$ with

$$
\begin{gathered}
\boldsymbol{\xi}_{1}(x)=\frac{1}{4 \pi} \operatorname{rot} \int_{\Omega} \frac{\boldsymbol{k}(y)-\operatorname{rota}^{*}(y)}{|x-y|} d y, \\
\left\{\begin{array}{l}
\nabla^{2} \omega(x)=0, \quad x \in \mathcal{F}_{1} \cup \mathcal{F}_{2} \cup \mathcal{F}_{3}, \quad[\omega]=0, \quad\left[\mu \frac{\partial \omega}{\partial N}\right]=-[\mu] \boldsymbol{\xi}_{1} \cdot \boldsymbol{N}, \quad x \in \mathcal{G}, \\
{[\omega]=0, \quad\left[\mu \frac{\partial \omega}{\partial n}\right]=-[\mu] \boldsymbol{\xi}_{1} \cdot \boldsymbol{n}, \quad x \in S_{3}, \quad \frac{\partial \omega}{\partial n}=-\boldsymbol{\xi}_{1} \cdot \boldsymbol{n}, \quad y \in S .}
\end{array}\right.
\end{gathered}
$$

The constants $c_{j}$ are found from the orthogonality conditions (3.14):

$$
0=\int_{\Omega} \mu\left(\boldsymbol{a}^{*}+\nabla \psi+\boldsymbol{\xi}\right) \cdot \widetilde{\boldsymbol{u}}_{k} d x+\sum_{j=1}^{b_{1}(\Omega)} c_{j} \int_{\Omega} \mu \widetilde{\boldsymbol{u}}_{j} \cdot \widetilde{\boldsymbol{u}}_{k} d x,
$$

$k=1, \ldots, b_{1}(\Omega)$. It is clear that

$$
\sum_{i=1}^{b_{1}}\left|c_{i}\right| \leqslant c\left\|\boldsymbol{a}^{*}+\nabla \psi+\boldsymbol{\xi}\right\|_{L_{2}(\Omega)}
$$

which implies

$$
\sum_{i=1}^{3}\left\|\sum_{j=1}^{b_{1}} c_{j} \widetilde{\boldsymbol{u}}_{j}\right\|_{W_{2}^{l+2}\left(\mathcal{F}_{i}\right)} \leqslant c\left\|\boldsymbol{a}^{*}+\nabla \psi+\boldsymbol{\xi}\right\|_{L_{2}(\Omega)} .
$$

Making use of well known estimates of the Newtonian potential and of solutions of the elliptic boundary value problems (3.20) and (3.22), we obtain

$$
\begin{aligned}
& \sum_{i=1}^{3}\|\nabla \psi\|_{W_{2}^{l+2}\left(\mathcal{F}_{i}\right)} \leqslant c\left\|\boldsymbol{a}^{*}\right\|_{W_{2}^{l+2}\left(\mathcal{F}_{1}\right)} \leqslant c\|\boldsymbol{a}\|_{W_{2}^{l+3 / 2}(\mathcal{G})}, \\
& \sum_{i=1}^{3}\|\boldsymbol{\xi}\|_{W_{2}^{l+2}\left(\mathcal{F}_{i}\right)} \leqslant c \sum_{i=1}^{3}\left\|\boldsymbol{k}-\operatorname{rot} \boldsymbol{a}^{*}\right\|_{W_{2}^{l+1}\left(\mathcal{F}_{i}\right)} \leqslant c\left(\sum_{i=1}^{3}\|\boldsymbol{k}\|_{W_{2}^{l+1}\left(\mathcal{F}_{i}\right)}+\|\boldsymbol{a}\|_{W_{2}^{l+3 / 2}(\mathcal{G})}\right) .
\end{aligned}
$$

Together with (3.24), these inequalities yield (3.15) The uniqueness follows from the fact that the solution of a homogeneous problem (3.4) belongs to $\widetilde{U}_{n}(\Omega)$.

Now we prove (3.17). We use the following representation formula for $\boldsymbol{h}$ :

$$
\boldsymbol{h}=\boldsymbol{A}+\nabla \Psi+\boldsymbol{X}+\sum_{j=1}^{b_{1}(\Omega)} c_{j} \widetilde{\boldsymbol{u}}_{j}
$$

where $\Psi$ and $\boldsymbol{X}$ are solutions to the problems

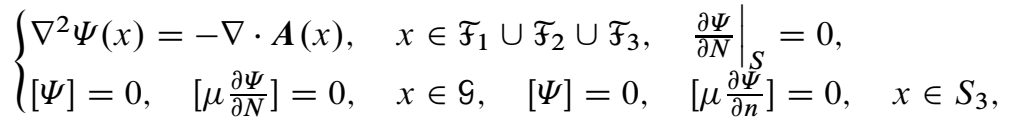

$$
\begin{aligned}
& \left\{\begin{array}{l}
\operatorname{rot} \boldsymbol{X}=\operatorname{rot} \boldsymbol{k}-\operatorname{rot} \boldsymbol{A}(x), \quad \nabla \cdot \boldsymbol{X}=0, \quad x \in \mathcal{F}_{1} \cup \mathcal{F}_{2} \cup \mathcal{F}_{3},\left.\quad \boldsymbol{X} \cdot \boldsymbol{n}\right|_{S}=0, \\
{[\mu \boldsymbol{X} \cdot \boldsymbol{N}]=0, \quad\left[\boldsymbol{X}_{\tau}\right]=0, \quad x \in \mathcal{G}, \quad[\mu \boldsymbol{X} \cdot \boldsymbol{n}]=0, \quad\left[\boldsymbol{X}_{\tau}\right]=0, \quad x \in S_{3} .}
\end{array}\right.
\end{aligned}
$$


Since $\left.\boldsymbol{A}\right|_{S}=0,\left.[\mu \boldsymbol{A} \cdot \boldsymbol{N}]\right|_{S}=0,\left.[\mu \boldsymbol{A} \cdot \boldsymbol{n}]\right|_{S_{3}}=0$, the problem (3.25) is solvable, and

$$
\|\nabla \Psi\|_{L_{2}(\Omega)} \leqslant c\|\boldsymbol{A}\|_{L_{2}(\Omega)} .
$$

As for $\boldsymbol{X}$, we represent it as the sum

$$
\begin{gathered}
\boldsymbol{X}(x)=\boldsymbol{X}_{1}(x)+\nabla U(x), \\
\boldsymbol{X}_{1}(x)=\frac{1}{4 \pi} \operatorname{rot} \int_{\Omega} \frac{\operatorname{rot}(\boldsymbol{K}(y)-\boldsymbol{A}(y))}{|x-y|} d y, \\
\left\{\begin{array}{l}
\nabla^{2} U(x)=0, \quad x \in \mathcal{F}_{1} \cup \mathcal{F}_{2} \cup \mathcal{F}_{3}, \quad[U(x)]=0, \quad\left[\mu \frac{\partial U}{\partial N}\right]=-[\mu] \boldsymbol{X}_{1} \cdot \boldsymbol{N}, \quad x \in \mathcal{G}, \\
{[U(x)]=0, \quad\left[\mu \frac{\partial U}{\partial n}\right]=-[\mu] \boldsymbol{X}_{1} \cdot \boldsymbol{n}, \quad x \in S_{3},\left.\quad \frac{\partial U}{\partial n}\right|_{S}=-\boldsymbol{X}_{1} \cdot \boldsymbol{n} .}
\end{array}\right.
\end{gathered}
$$

Since $\left.[\boldsymbol{N} \times(\boldsymbol{K}-\boldsymbol{A})]\right|_{\mathrm{g}}=\boldsymbol{N} \times\left.\left(\left[\boldsymbol{K}_{\tau}\right]-\boldsymbol{a}\right)\right|_{\mathrm{g}}=0$, we have $\boldsymbol{X}_{1}(x)=\frac{1}{4 \pi} \operatorname{rot} \int_{\Omega} \nabla \frac{1}{|x-y|} \times(\boldsymbol{K}-\boldsymbol{A}) d y$, and by the Calderon-Zygmund theorem

$$
\left\|\boldsymbol{X}_{1}\right\|_{L_{2}(\Omega)} \leqslant c\left(\|\boldsymbol{K}\|_{L_{2}(\Omega)}+\|\boldsymbol{A}\|_{L_{2}(\Omega)}\right)
$$

In addition,

$$
\begin{aligned}
\|\nabla U\|_{L_{2}(\Omega)} & \leqslant c\left(\left\|\boldsymbol{X}_{1} \cdot \boldsymbol{N}\right\|_{W_{2}^{-1 / 2}(\mathcal{})}+\left\|\boldsymbol{X}_{1} \cdot n\right\|_{W_{2}^{-1 / 2}\left(S_{3}\right)}\right) \\
& \leqslant c\left\|\boldsymbol{X}_{1}\right\|_{L_{2}(\Omega)},
\end{aligned}
$$

because $X_{1}$ is divergence free.

Putting the inequalities (3.26)-(3.28) together, we obtain (3.17). The theorem is proved.

Corollary 1 Assume that $\boldsymbol{k}, \boldsymbol{K}, \boldsymbol{a}, \boldsymbol{A}$ in (3.4), (3.16) depend on $t \in(0, T), \boldsymbol{k} \in W_{2}^{l+1,0}\left(Q_{T}^{j}\right)$, $\boldsymbol{k}(\cdot, t) \in W_{2}^{l}\left(\mathcal{F}_{j}\right), j=1,2,3, \boldsymbol{a} \in W_{2}^{l+3 / 2,0}\left(G_{T}\right), \boldsymbol{a}(\cdot, t) \in W_{2}^{l+1 / 2}(\mathcal{G}) \forall t<T, \boldsymbol{K}, \boldsymbol{A} \in$ $W_{2}^{0,1+l / 2}\left(Q_{T}\right)$. Then

$$
\begin{aligned}
& \sum_{j=1}^{3}\left\|\boldsymbol{h}^{(j)}\right\|_{H^{l+2, l / 2+1}\left(Q_{T}^{j}\right)} \\
& \leqslant c\left(\sum_{i=1}^{3}\left(\left\|\boldsymbol{k}^{(i)}\right\|_{W_{2}^{l+1,0}\left(Q_{T}^{i}\right)}+\sup _{t<T}\left\|\boldsymbol{k}^{(i)}(\cdot, t)\right\|_{W_{2}^{l}\left(\mathcal{F}_{i}\right)}+\|\boldsymbol{K}\|_{\widehat{W}_{2}^{0,1+l / 2}\left(Q_{T}\right)}\right)\right) \\
& +\|\boldsymbol{a}\|_{W_{2}^{l+3 / 2,0}\left(G_{T}\right)}+\sup _{t<T}\|\boldsymbol{a}\|_{W_{2}^{l+1 / 2}(\mathcal{S})} \\
& +\|\boldsymbol{A}\|_{\widehat{W}_{2}^{0,1+l / 2}\left(Q_{T}\right)}
\end{aligned}
$$

Estimate (3.29) follows from (3.15) and from (3.17) applied to the time derivative $\boldsymbol{h}_{t}$ and to the finite difference of the time derivative $\boldsymbol{h}_{t}(x, t+s)-\boldsymbol{h}_{t}(x, t)$ (cf. the analogous corollary in [20]).

Let us pass to the problem 2. (3.2), (3.3).

THEOREM 4 Assume that $\boldsymbol{G}_{1} \in W_{2}^{l+1,0}\left(Q_{T}^{i}\right) \cap W_{2}^{l / 2}\left(0, T ; W_{2}^{1}\left(\mathcal{F}_{i}\right)\right), i=1,3, \boldsymbol{G}_{2} \in W_{2}^{l, l / 2}\left(Q_{T}^{i}\right)$, $\boldsymbol{H}_{0} \in W_{2}^{l+1}\left(\mathcal{F}_{i}\right), i=1,2,3, \ell \in W_{2}^{l+2, l / 2+1}\left(Q_{T}^{2}\right),\left.\ell(y, t)\right|_{y \in S_{3} \cup S}=0, \boldsymbol{a} \in W_{2}^{l+3 / 2,0}\left(G_{T}\right)$, 
$\boldsymbol{a}(\cdot, t) \in W_{2}^{l+1 / 2}(\mathcal{G}), \forall t \in(0, T), \boldsymbol{a}=[\boldsymbol{A}], \boldsymbol{A}_{t} \in W_{2}^{0, l / 2}\left(Q_{T}\right)$ and that the compatibility conditions

$$
\begin{aligned}
& \nabla \cdot \boldsymbol{H}_{0}(y)=0, \quad y \in \mathcal{F}_{1} \cup \mathcal{F}_{2} \cup \mathcal{F}_{3}, \quad \operatorname{rot} \boldsymbol{H}_{0}(y)=\operatorname{rot} \ell(y, 0), \quad y \in \mathcal{F}_{2}, \\
& {\left[\mu \boldsymbol{H}_{0} \cdot \boldsymbol{N}\right]=0, \quad\left[\boldsymbol{H}_{0 \tau}\right]=\left(\left.\frac{\widehat{\mathcal{L}} \widehat{\mathcal{L}}^{T} \boldsymbol{N}}{\left|\widehat{\mathcal{L}}^{T} \boldsymbol{N}\right|^{2}} \boldsymbol{N}\right|_{\rho=\rho_{0}}-\boldsymbol{N}\right)\left[\boldsymbol{H}_{0} \cdot \boldsymbol{N}\right],} \\
& {\left[\mu \boldsymbol{H}_{0} \cdot \boldsymbol{n}\right]=0, \quad\left[\boldsymbol{H}_{0 \tau}\right]=0, \quad y \in S_{3}, \quad \boldsymbol{H}_{0} \cdot \boldsymbol{n}=0, \quad y \in S .}
\end{aligned}
$$

are satisfied. Then the problem 2. has a unique solution $\boldsymbol{H} \in W_{2}^{l+2, l / 2+1}\left(Q_{T}^{i}\right), i=1,2,3$, and

$$
\begin{aligned}
& \sum_{i=1}^{3}\|\boldsymbol{H}\|_{H^{l+2, l / 2+1}\left(Q_{T}^{i}\right)} \\
& \leqslant c\left(\sum_{j=1,3}\left(\left\|\boldsymbol{G}_{1}\right\|_{W_{2}^{l+1,0}\left(Q_{T}^{j}\right)}+\left\|\boldsymbol{G}_{1}\right\|_{\widehat{W}_{2}^{l / 2}\left(0, T ; W_{2}^{1}\left(\mathcal{F}_{j}\right)\right)}\right)+\sum_{i=1}^{3}\left\|\boldsymbol{G}_{2}\right\|_{\widehat{W}_{2}^{l, l / 2}\left(Q_{T}^{i}\right)}\right. \\
& +\|\boldsymbol{\ell}\|_{W_{2}^{l+2,0}\left(Q_{T}^{2}\right)}+\sup _{t<T}\|\boldsymbol{\ell}(\cdot, t)\|_{W_{2}^{l+1}\left(\mathcal{F}_{2}\right)}+\|\boldsymbol{\ell}\|_{\widehat{W}_{2}^{0, l+l / 2}\left(Q_{T}^{2}\right)} \\
& \left.+\|\boldsymbol{a}\|_{W_{2}^{l+3 / 2,0}\left(G_{T}\right)}+\sup _{t<T}\|\boldsymbol{a}(\cdot, t)\|_{W_{2}^{l+1 / 2}(\mathcal{G})}+\|\boldsymbol{A}\|_{\widehat{W}_{2}^{0,1+l / 2}\left(Q_{T}\right)}+\sum_{i=1}^{3}\left\|\boldsymbol{H}_{0}\right\|_{W_{2}^{l+1}\left(\mathfrak{F}_{i}\right)}\right) .
\end{aligned}
$$

The solution is unique in the class of vector fields with the above-mentioned regularity properties that are orthogonal to $\widetilde{U}_{n}(\Omega)$ :

$$
\int_{\Omega} \mu \boldsymbol{H} \cdot \widetilde{\boldsymbol{u}}_{k}(y) d y=0, \quad k=1, \ldots, b_{1}(\Omega)
$$

Proof. Step 1. Reduction to the case $\ell=0, a=0$.

We extend $\ell$ from $\mathcal{F}_{2}$ in $\mathcal{F}_{1}$ in such a way that the extension $\ell^{*}$ satisfies

$$
\begin{aligned}
& \left\|\ell^{*}\right\|_{W_{2}^{l+2}\left(\mathcal{F}_{1} \cup \mathcal{F}_{2}\right)} \leqslant c\left\|\ell^{*}\right\|_{W_{2}^{l+2}\left(\mathcal{F}_{2}\right)}, \\
& \|\ell\|_{L_{2}\left(\mathcal{F}_{1} \cup \mathcal{F}_{2}\right)} \leqslant c\|\ell\|_{L_{2}\left(\mathcal{F}_{2}\right)},
\end{aligned}
$$

then

$$
\left\|\ell^{*}\right\|_{W_{2}^{l+2,0}\left(Q_{T}^{1} \cup Q_{T}^{2}\right)}+\left\|\ell^{*}\right\|_{\widehat{W}_{2}^{0,1+l / 2}\left(Q_{T}^{1} \cup Q_{T}^{2}\right)} \leqslant c\left(\|\ell\|_{W_{2}^{l+2,0}\left(Q_{T}^{2}\right)}+\|\ell\|_{\widehat{W}_{2}^{0,1+l / 2}\left(Q_{T}^{2}\right)}\right) .
$$

For $y \in \mathcal{F}_{3}$ we set $\ell(y, t)=0$. We define $\boldsymbol{h}_{1}$ as a solution of the problem (3.4) with $\boldsymbol{k}=\operatorname{rot}\left(\boldsymbol{\ell}^{*}+\right.$ $A$ ); it satisfies the inequality (3.29) with $\boldsymbol{K}=\ell^{*}+A$. For $\boldsymbol{h}=\boldsymbol{H}-\boldsymbol{h}_{1}$ we obtain the problem 2. with $\boldsymbol{\ell}=0, \boldsymbol{a}=0$ and with $\boldsymbol{G}_{1}, \boldsymbol{G}_{2}$ replaced by $\boldsymbol{G}_{1}^{\prime}=\boldsymbol{G}_{1}-\alpha^{-1} \operatorname{roth}_{1}, \boldsymbol{G}_{2}^{\prime}=\boldsymbol{G}_{2}-\boldsymbol{h}_{1 t}$.

Step 2. Proof of the solvability of the Problem 2 with $\ell=0, a=0$. 
This problem can be written in the form

$$
\left\{\begin{array}{l}
\int_{0}^{T} \int_{\Omega} \mu \boldsymbol{h}_{t} \cdot \boldsymbol{\psi}(y, t) d y d t+\int_{0}^{T} \int_{\mathcal{F}_{1} \cup \mathcal{F}_{3}} \alpha^{-1} \operatorname{roth} \cdot \operatorname{rot} \boldsymbol{\psi}(y, t) d y d t \\
=\int_{0}^{T} \int_{\mathcal{F}_{1} \cup \mathcal{F}_{3}} \boldsymbol{g}_{1}(y, t) \cdot \operatorname{rot} \boldsymbol{\psi}(y, t) d y d t+\int_{0}^{T} \int_{\Omega} \mu \boldsymbol{g}_{2}(y, t) \cdot \boldsymbol{\psi}(y, t) d y d t, \\
\nabla \cdot \boldsymbol{h}(y, t)=0, \quad y \in \mathcal{F}_{1} \cup \mathcal{F}_{2} \cup \mathcal{F}_{3}, \quad \operatorname{roth}(y, t)=0, \quad y \in \mathcal{F}_{2}, \\
{[\mu \boldsymbol{h} \cdot \boldsymbol{N}]=0, \quad\left[\boldsymbol{h}_{\tau}\right]=0, \quad y \in \mathcal{G},} \\
{[\mu \boldsymbol{h} \cdot \boldsymbol{n}]=0, \quad\left[\boldsymbol{h}_{\tau}\right]=0, \quad y \in S_{3}, \quad \boldsymbol{h} \cdot \boldsymbol{n}=0, \quad y \in S,} \\
\boldsymbol{h}(y, 0)=\boldsymbol{H}_{0}(y)-\boldsymbol{h}_{1}(y, 0) \equiv \boldsymbol{h}_{0}(y),
\end{array}\right.
$$

where

$$
\boldsymbol{g}_{1}=P_{J} \boldsymbol{G}_{1}^{\prime}, \quad \boldsymbol{g}_{2}=P_{\mathcal{H}} P_{\mu} \boldsymbol{G}_{2}^{\prime},
$$

$P_{J}$ is the orthogonal projection on the space $\stackrel{\circ}{J}\left(\mathcal{F}_{1} \cup \mathcal{F}_{3}\right)$.

Now we decompose $\boldsymbol{g}_{2}$ and $\boldsymbol{h}$ in the sum of linear combinations of the modified Neumann vector fields $\widetilde{\boldsymbol{u}}_{q}(x)$ in $\Omega$ and of the vector fields that are orthogonal to $\widetilde{U}_{n}(\Omega)$. We set

$$
\boldsymbol{h}=\boldsymbol{h}^{\prime}+\boldsymbol{h}^{\prime \prime}, \quad \boldsymbol{g}_{2}=\boldsymbol{g}_{2}^{\prime}+\boldsymbol{g}_{2}^{\prime \prime},
$$

where

$$
\boldsymbol{h}^{\prime}=P_{\mathcal{H}_{\perp}} \boldsymbol{h}, \quad \boldsymbol{g}_{2}^{\prime}=P_{\mathcal{H}_{\perp}} \boldsymbol{g}_{2}, \quad \boldsymbol{h}^{\prime \prime}(y, t)=\sum_{j=1}^{b_{1}(\Omega)} x_{j}(t) \widetilde{\boldsymbol{u}}_{j}(y), \quad \boldsymbol{g}_{2}^{\prime \prime}(y, t)=\sum_{j=1}^{b_{1}(\Omega)} \gamma_{j}(t) \widetilde{\boldsymbol{u}}_{j}(y) .
$$

In the same way we decompose the test function $\boldsymbol{\psi}$ and the initial datum $\boldsymbol{h}_{0}$. Then (3.33) is decomposed in two problems. Setting $\boldsymbol{\psi}=\boldsymbol{\psi}^{\prime \prime}$, we reduce (3.33) to

$$
\boldsymbol{h}_{t}^{\prime \prime}=\boldsymbol{g}_{2}^{\prime \prime}, \quad \boldsymbol{h}^{\prime \prime}(y, 0)=\boldsymbol{h}_{0}^{\prime \prime}(y),
$$

and for $\boldsymbol{h}^{\prime}$ we obtain the problem

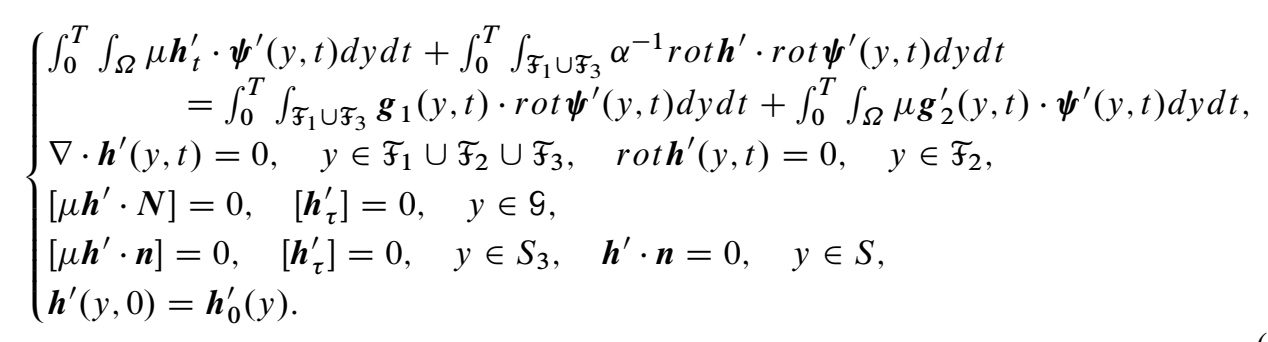

Clearly, (3.34) is easily solved. We have

$$
\boldsymbol{h}^{\prime \prime}(y, t)=\boldsymbol{h}_{0}^{\prime \prime}(y)+\int_{0}^{t} \boldsymbol{g}_{2}^{\prime \prime}(y, \tau) d \tau
$$


which is equivalent to

$$
x_{j}(t)=x_{j}(0)+\int_{0}^{t} \gamma_{j}(\tau) d \tau, \quad j=1, \ldots, b_{1}(\Omega) .
$$

As for (3.35), this problem is studied in [17]. Indeed, let $E$ be the extension operator defined on the space $J\left(\mathcal{F}_{1} \cup \mathcal{F}_{3}\right) \cap W_{2}^{1}\left(\mathcal{F}_{1} \cup \mathcal{F}_{3}\right)$ such that $\mathrm{E} \psi \in W_{2}^{1}(\Omega)$ and $(\mathrm{E} \psi)_{\tau}=0$ on $S$. Then

$$
\begin{gathered}
\int_{\mathcal{F}_{1} \cup \mathcal{F}_{3}} \boldsymbol{g}_{1} \cdot \operatorname{rot} \boldsymbol{\psi}^{\prime} d y=\int_{\Omega} \mathrm{Eg}_{1} \cdot \operatorname{rot} \boldsymbol{\psi}^{\prime} d y=\int_{\Omega} \operatorname{rot} \mathrm{E}_{1} \cdot \boldsymbol{\psi}^{\prime} d y=\int_{\Omega} \mu P_{\mathcal{H}_{\perp}} \mu^{-1} \operatorname{rot} \mathrm{E} \boldsymbol{g}_{1} \cdot \boldsymbol{\psi}^{\prime} d y, \\
\int_{\mathcal{F}_{1} \cup \mathcal{F}_{3}} \alpha^{-1} \operatorname{roth}^{\prime} \cdot \operatorname{rot} \boldsymbol{\psi}^{\prime}(y, t) d y=\int_{\Omega} \mu P_{\mathcal{H}_{\perp}}(\mu \alpha)^{-1} \operatorname{rot}(\text { Eroth }) \cdot \operatorname{rot} \boldsymbol{\psi}^{\prime} d y .
\end{gathered}
$$

Consequently (3.35) can be written as the Cauchy problem

$$
\boldsymbol{h}_{t}^{\prime}+Q \boldsymbol{h}^{\prime}=\boldsymbol{g}_{2}^{\prime}+\boldsymbol{g}_{3} \equiv g_{4},\left.\quad \boldsymbol{h}^{\prime}\right|_{t=0}=\boldsymbol{h}_{0}^{\prime}
$$

where

and

$$
\boldsymbol{g}_{3}=P_{\mathcal{H}_{\perp}} \mu^{-1} \operatorname{rot} \mathrm{Eg}_{1}
$$

$$
Q \boldsymbol{h}^{\prime}=P_{\mathcal{H}_{\perp}} \mu^{-1} \operatorname{rot} \mathrm{E} \alpha^{-1} \operatorname{rot} \boldsymbol{h}^{\prime} .
$$

The operator $Q$ is a positive operator defined on the space of vector fields $\boldsymbol{h} \in W_{2}^{2}\left(\mathcal{F}_{1} \cup \mathcal{F}_{2} \cup \mathcal{F}_{3}\right) \cup$ $\mathcal{H}^{2}(\Omega)$ (see details in [17]). Now we pass to the proof of (3.31). It is clear that

$$
\begin{aligned}
& \sum_{i=1}^{3}\left\|\boldsymbol{h}^{\prime \prime}\right\|_{H^{l+2, l / 2+1}\left(Q_{T}^{i}\right)} \\
& \leqslant c(T)\left(\|\boldsymbol{x}\|_{\widehat{W}_{2}^{1+l / 2}(0, T)}+\left|\boldsymbol{x}_{0}\right|+\|\boldsymbol{\gamma}\|_{\left.\widehat{W}_{2}^{l / 2}(0, T)\right)}\right) \\
& \leqslant c(T) \sum_{i=1}^{3}\left(\left\|\boldsymbol{h}_{0}\right\|_{W_{2}^{l+1}\left(\mathfrak{F}_{i}\right)}+\left\|\boldsymbol{g}_{2}\right\|_{\widehat{W}_{2}^{l, l / 2}\left(Q_{T}^{i}\right)}\right),
\end{aligned}
$$

because

$$
\begin{aligned}
& \int_{\Omega} \mu \boldsymbol{h}_{0}(y) \cdot \widetilde{\boldsymbol{u}}_{k}(y) d y=\int_{\Omega} \mu \boldsymbol{h}_{0}^{\prime \prime}(y) \cdot \widetilde{\boldsymbol{u}}_{k}(y) d y=\sum_{q=1}^{b_{1}(\Omega)} x_{q}(0) \int_{\Omega} \mu \widetilde{\boldsymbol{u}}_{k}(y) \cdot \widetilde{\boldsymbol{u}}_{q}(y) d y, \\
& \int_{\Omega} \mu \boldsymbol{g}_{2}(y, t) \cdot \widetilde{\boldsymbol{u}}_{k}(y) d y=\sum_{q=1}^{b_{1}(\Omega)} \gamma_{q}(t) \int_{\Omega} \mu \widetilde{\boldsymbol{u}}_{k}(y) \cdot \widetilde{\boldsymbol{u}}_{q}(y) d y
\end{aligned}
$$

Next, we estimate the functions $\boldsymbol{g}_{2}^{\prime}$ and $\boldsymbol{g}_{3}$ in (3.37) making use of the boundedness of the projection operators $P_{J}, P_{\mu}, P_{\mathcal{H}}, P_{\mathcal{H}_{\perp}}$ :

$$
\begin{gathered}
\left\|P_{J} \boldsymbol{G}_{1}^{\prime}\right\|_{W_{2}^{r}\left(\mathcal{F}_{i}\right)} \leqslant c\left\|\boldsymbol{G}_{1}^{\prime}\right\|_{W_{2}^{r}\left(\mathcal{F}_{i}\right)}, \quad i=1,3, \quad r=1, \quad r=l+1, \\
\left\|P_{\mu} \boldsymbol{G}_{2}^{\prime}\right\|_{L_{2}(\Omega)} \leqslant c\left\|\boldsymbol{G}_{2}^{\prime}\right\|_{L_{2}(\Omega)}, \quad \sum_{i=1}^{3}\left\|P_{\mu} \boldsymbol{G}_{2}^{\prime}\right\|_{W_{2}^{l}\left(\mathfrak{F}_{i}\right)} \leqslant c\left\|\boldsymbol{G}_{2}^{\prime}\right\|_{W_{2}^{l}\left(\mathcal{F}_{i}\right)} ;
\end{gathered}
$$


moreover we require that

$$
\|\mathrm{E} \boldsymbol{f}\|_{W_{2}^{r}(\Omega)} \leqslant c \sum_{i=1,3}\|\boldsymbol{f}\|_{W_{2}^{r}\left(\mathcal{F}_{i}\right)}, \quad r=1, l+1 .
$$

This implies

$$
\begin{gathered}
\sum_{i=1}^{3}\left\|\boldsymbol{g}_{3}\right\|_{\widehat{W}_{2}^{l, l / 2}\left(Q_{T}^{i}\right)} \leqslant c \sum_{i=1,3}\left(\left\|\boldsymbol{g}_{1}\right\|_{W_{2}^{l+1,0}\left(Q_{T}^{i}\right)}+\left\|\boldsymbol{g}_{1}\right\|_{\widehat{W}_{2}^{l / 2}\left(0, T: W_{2}^{1}\left(\mathcal{F}_{i}\right)\right)}\right) \\
\leqslant c \sum_{i=1,3}\left(\left\|\boldsymbol{G}_{1}^{\prime}\right\|_{W_{2}^{l+1,0}\left(Q_{T}^{i}\right)}+\left\|\boldsymbol{G}_{1}^{\prime}\right\|_{\widehat{W}_{2}^{l / 2}\left(0, T: W_{2}^{1}\left(\mathcal{F}_{i}\right)\right)}\right) \\
\sum_{i=1,3}\left\|\boldsymbol{g}_{2}^{\prime}\right\|_{\widehat{W}_{2}^{l, l / 2}\left(Q_{T}^{i}\right)} \leqslant c \sum_{i=1,3}\left\|\boldsymbol{G}_{2}^{\prime}\right\|_{\widehat{W}_{2}^{l, l / 2}\left(Q_{T}^{i}\right)} .
\end{gathered}
$$

Hence

$$
\begin{aligned}
& \sum_{i=1}^{3}\left\|\boldsymbol{g}_{4}\right\|_{\widehat{W}_{2}^{l, l / 2}\left(Q_{T}^{i}\right)} \\
& \quad \leqslant c\left(\sum_{i=1}^{3}\left\|\boldsymbol{G}_{2}^{\prime}\right\|_{\widehat{W}_{2}^{l, l / 2}\left(Q_{T}^{i}\right)}+\sum_{i=1,3}\left(\left\|\boldsymbol{G}_{1}^{\prime}\right\|_{W_{2}^{l+1,0}\left(Q_{T}^{i}\right)}+\left\|\boldsymbol{G}_{1}^{\prime}\right\|_{\widehat{W}_{2}^{l / 2}\left(0, T ; W_{2}^{1}\left(\mathcal{F}_{i}\right)\right.}\right)\right)
\end{aligned}
$$

Now we make use of the following result.

THEOREM 5 For arbitrary $\boldsymbol{g}_{4} \in \cap_{i=1}^{3} W_{2}^{l, l / 2}\left(Q_{T}^{i}\right) \cap L_{2}\left(0, T ; \mathcal{H}_{\perp}^{l}(\Omega)\right)$ and $\boldsymbol{h}_{0}^{\prime} \in \mathcal{H}_{\perp}^{l+1}(\Omega)$ the problem (3.37) has a unique solution

$$
\boldsymbol{h}^{\prime} \in \cap_{i=1}^{3} W_{2}^{2+l, 1+l / 2}\left(Q_{T}^{i}\right) \cap L_{2}\left(0, T ; \mathcal{H}_{\perp}^{1+2}(\Omega)\right)
$$

and

$$
\sum_{i=1}^{3}\left\|\boldsymbol{h}^{\prime}\right\|_{H^{2+l, 1+l / 2}\left(Q_{T}^{i}\right)} \leqslant c \sum_{i=1}^{3}\left(\left\|\boldsymbol{g}_{4}\right\|_{\widehat{W}_{2}^{l, l / 2}\left(Q_{T}^{i}\right)}+\left\|\boldsymbol{h}_{0}^{\prime}\right\|_{W_{2}^{l+1}\left(\mathcal{F}_{i}\right)}\right) .
$$

This result is obtained in [17] for $l=0, \mathcal{F}_{3}=\emptyset$, and in [20] it is extended to $l \in(1 / 2,1)$ in the case of simply connected $\mathcal{F}_{1}$ and $\Omega$, when $\widetilde{U}_{n}(\Omega)=\emptyset$. The general case is considered in a similar manner.

The inequality (3.31) for the solution of the Problem 2 is a consequence of (3.29), (3.40)-(3.43). The uniqueness follows from the uniqueness of the solution of (3.37). Theorem 4 is proved. 


\section{Nonlinear problem}

In this section we consider the main nonlinear problem

$$
\left\{\begin{array}{l}
\boldsymbol{u}_{t}-\rho_{t}^{*}\left(\mathcal{L}^{-1} \boldsymbol{N}^{*}(y) \cdot \nabla\right) \boldsymbol{u}+\left(\mathcal{L}^{-1} \boldsymbol{u} \cdot \nabla\right) \boldsymbol{u} \\
-\widetilde{\nabla} \cdot \widetilde{T}(\boldsymbol{u}, q)-\widetilde{\nabla} \cdot T_{M}\left(\frac{\mathcal{L}}{L} \boldsymbol{h}\right)=\boldsymbol{f}\left(e_{\rho}, t\right), \\
\nabla \cdot \widehat{\mathcal{L}} \boldsymbol{u}=0, \quad y \in \mathcal{F}_{1}, \quad t>0, \\
\widetilde{T}(\boldsymbol{u}, q) \boldsymbol{n}\left(e_{\rho}\right)+\left[T_{M}\left(\frac{\mathcal{L}}{L} \boldsymbol{h}\right) \boldsymbol{n}\right]=\sigma H\left(e_{\rho}\right) \boldsymbol{n}, \quad \rho_{t}=\frac{\boldsymbol{u} \cdot \widehat{\mathcal{L}}^{T} \boldsymbol{N}}{\Lambda(y, \rho)}, \quad y \in \mathcal{G}, \\
\int_{0}^{T} \int_{\Omega} \mu\left(\boldsymbol{h}_{t}-\boldsymbol{\Phi}(\boldsymbol{h}, \rho)\right) \cdot \boldsymbol{\psi}(y, t) d y d t+\int_{0}^{T} \int_{\mathcal{F}_{1} \cup \mathcal{F}_{3}} \alpha^{-1} \operatorname{Prot} \operatorname{Ph} \cdot \operatorname{rot} \boldsymbol{\psi}(y, t) d y d t \\
-\mu_{1} \int_{0}^{T} \int_{\mathcal{F}_{1}}\left(\mathcal{L}^{-1} \boldsymbol{u} \times \boldsymbol{h}\right) \cdot \operatorname{rot} \boldsymbol{\psi}(y, t) d y d t+\int_{0}^{T} d t \int_{\mathcal{G}}^{\boldsymbol{\Psi}}(\boldsymbol{u}, \boldsymbol{h}, \rho) \cdot \boldsymbol{\psi} d S d t \\
=\int_{0}^{T} \int_{\mathcal{F}_{3}} \alpha^{-1} \boldsymbol{j}(y, t) \cdot \operatorname{rot} \boldsymbol{\psi}(y, t) d y d t, \\
\nabla \cdot \boldsymbol{h}(y, t)=0, \quad y \in \mathcal{F}_{1} \cup \mathcal{F}_{2} \cup \mathcal{F}_{3}, \quad \operatorname{rot} \mathbb{P} \boldsymbol{h}(y, t)=0, \quad y \in \mathcal{F}_{2}, \\
{[\mu \boldsymbol{h} \cdot \boldsymbol{N}]=0, \quad\left[\boldsymbol{h}_{\tau}\right]=\left(\frac{\widehat{\mathcal{L}}^{T} \widehat{\mathcal{L}}^{T}}{\left|\widehat{\mathcal{L}}^{T} \boldsymbol{N}\right|^{2}}-\boldsymbol{N}\right)[\boldsymbol{h} \cdot \boldsymbol{N}], \quad y \in \mathcal{G},} \\
{[\mu \boldsymbol{h} \cdot \boldsymbol{n}]=0, \quad\left[\boldsymbol{h}_{\tau}\right]=0, \quad y \in S_{3}, \quad \boldsymbol{h} \cdot \boldsymbol{n}=0, \quad y \in S,} \\
\boldsymbol{u}(y, 0)=\boldsymbol{u}_{0}(y), \quad y \in \mathcal{F}_{1}, \quad \boldsymbol{h}(y, 0)=\boldsymbol{h}_{0}(y), \quad y \in \mathcal{F}_{1} \cup \mathcal{F}_{2} \cup \mathcal{F}_{3},
\end{array}\right.
$$

where $\psi$ is an arbitrary test function satisfying the conditions (2.22), (2.23).

As in [20], we transform the problem (4.1) by separating the linear and nonlinear parts with respect to $\boldsymbol{u}, q, \rho, \boldsymbol{h}$ in all the equations and separating the tangential and normal components in the equation $\widetilde{T}(\boldsymbol{u}, q) \boldsymbol{n}\left(e_{\rho}\right)=\sigma H\left(e_{\rho}\right) \boldsymbol{n}$. We also make use of the formulas

$$
\begin{aligned}
\boldsymbol{f}\left(e_{\rho}, t\right) & =\boldsymbol{f}(y, t)+\int_{0}^{1} \frac{d}{d s} \boldsymbol{f}\left(e_{s \rho}, t\right) d s=\boldsymbol{f}(y, t)+\left.\frac{d}{d s} \boldsymbol{f}\left(e_{s \rho}, t\right)\right|_{s=0} \\
+ & \int_{0}^{1}(1-s) \frac{d^{2}}{d s^{2}} \boldsymbol{f}\left(e_{s \rho}, t\right) d s=\boldsymbol{f}(y, t)+\rho^{*}(y, t)\left(\boldsymbol{N}^{*} \cdot \nabla\right) \boldsymbol{f}(y, t) \\
+ & \int_{0}^{1}(1-s) \frac{d^{2}}{d s^{2}} \boldsymbol{f}\left(e_{s \rho}, t\right) d s \\
H\left(e_{\rho}\right) & =-\left.\nabla_{x} \cdot \boldsymbol{n}(x)\right|_{x=e_{\rho}(y, t)}=-\mathcal{L}^{-T}(y, \rho) \nabla \cdot \frac{\mathcal{L}^{T}(y, \rho) \boldsymbol{N}(y)}{\left|\mathcal{L}^{T}(y, \rho) \boldsymbol{N}(y)\right|} \\
& =\mathcal{H}_{g}(y)-\mathfrak{B} \rho-\int_{0}^{1}(1-s) \frac{d^{2}}{d s^{2}} \mathcal{L}^{-T}(y, s \rho) \nabla \cdot \frac{\mathcal{L}^{T}(y, s \rho) \boldsymbol{N}(y)}{\left|\mathcal{L}^{T}(y, s \rho) \boldsymbol{N}(y)\right|}
\end{aligned}
$$

where $\mathfrak{B} \rho=-\Delta_{\mathrm{g}} \rho-\left(\mathcal{H}_{\mathrm{g}}^{2}-2 \mathcal{K}_{\mathrm{g}}\right) \rho$ is the first variation of $-\left(H\left(e_{\rho}\right)-\mathcal{H}_{\mathrm{g}}(y)\right)$ with respect to $\rho$.

Without restriction of generality, we assume that $\boldsymbol{f}(x, t)$ is given in the whole space $\mathbb{R}^{3}$. Since 
$\boldsymbol{\Psi} \cdot \boldsymbol{\psi}=\boldsymbol{\Psi}_{\tau} \cdot \boldsymbol{\psi}_{\tau}=(\boldsymbol{N} \times \boldsymbol{\Psi}) \cdot(\boldsymbol{N} \times \boldsymbol{\psi})$, the surface integral in (4.1) can be written in the form

$$
\int_{\mathcal{G}} \boldsymbol{\Psi} \cdot \boldsymbol{\psi}(y, t) d S=-\int_{\mathcal{F}_{1}} \operatorname{rot}\left(\boldsymbol{N}^{*} \times \boldsymbol{\Psi}^{*}\right) \cdot \boldsymbol{\psi}(y, t) d y+\int_{\mathcal{F}_{1}}\left(\boldsymbol{N}^{*} \times \boldsymbol{\Psi}^{*}\right) \cdot \operatorname{rot} \boldsymbol{\psi}(y, t) d y,
$$

where $N^{*}$ is the extension of $N$ in $\Omega$ mentioned in Sec.2 and

$$
\boldsymbol{\Psi}^{*}=\frac{\boldsymbol{u} \cdot \widehat{\mathcal{L}}^{T}\left(y, \rho^{*}\right) \boldsymbol{N}^{*}(y)}{J\left(y, \rho^{*}\right)}[\mu]\left(\boldsymbol{h}-\boldsymbol{N}^{*}\left(\boldsymbol{N}^{*} \cdot \boldsymbol{h}\right)\right), \quad y \in \mathcal{F}_{1} .
$$

Finally, we introduce the vector field $V \in W_{2}^{l+3 / 2}(\mathcal{G})$, satisfying the condition

$$
\left\|\boldsymbol{V}-\boldsymbol{u}_{0}\right\|_{W_{2}^{l+1 / 2}(\mathcal{G})} \leqslant \delta \ll 1
$$

It is easily seen that (4.1) is equivalent to

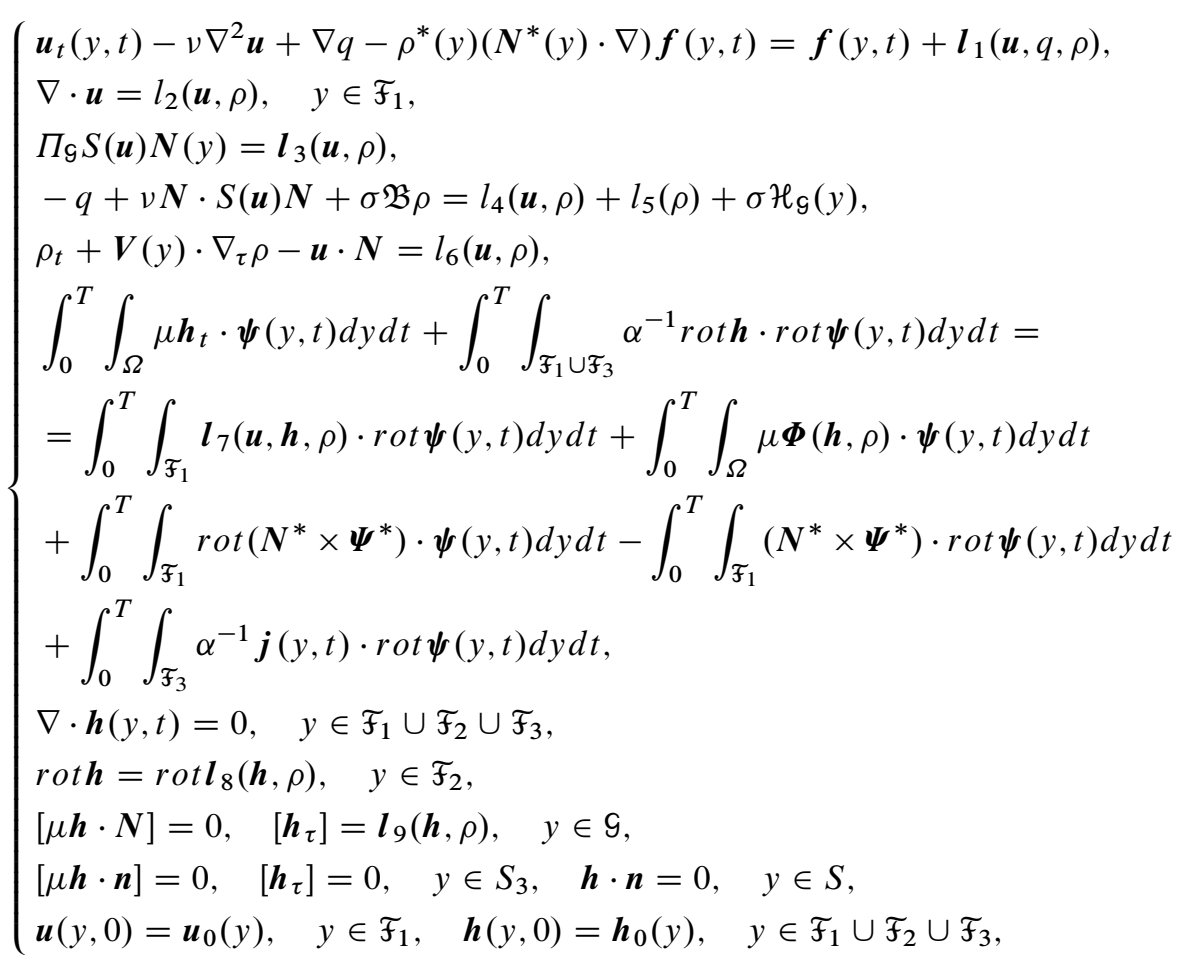


where

$$
\left\{\begin{array}{l}
\boldsymbol{l}_{1}(\boldsymbol{u}, q, \rho)=v\left(\widetilde{\nabla}^{2}-\nabla^{2}\right) \boldsymbol{u}+(\nabla-\widetilde{\nabla}) q+\rho_{t}^{*}\left(\mathcal{L}^{-1} \boldsymbol{N}^{*}(y) \cdot \nabla\right) \boldsymbol{u} \\
-\left(\mathcal{L}^{-1} \boldsymbol{u} \cdot \nabla\right) \boldsymbol{u}+\widetilde{\nabla} \cdot T_{M}\left(\frac{\mathcal{L}}{J} \boldsymbol{h}\right)+\int_{0}^{1}(1-s) \frac{d^{2}}{d s^{2}} \boldsymbol{f}\left(e_{s \rho}, t\right) d s, \\
l_{2}(\boldsymbol{u}, \rho)=\left(I-\widehat{\mathcal{L}}^{T}\right) \nabla \cdot \boldsymbol{u}=\nabla \cdot \boldsymbol{L}(\boldsymbol{u}, \rho), \\
\boldsymbol{L}(\boldsymbol{u}, \rho)=(I-\widehat{\mathcal{L}}) \boldsymbol{u}, \quad y \in \mathcal{F}_{1}, \\
\boldsymbol{l}_{3}(\boldsymbol{u}, \rho)=\Pi_{\mathcal{G}}\left(\Pi_{\mathrm{g}} S(\boldsymbol{u}) \boldsymbol{N}\right)(y)-\Pi \widetilde{S}(\boldsymbol{u}) \boldsymbol{n}\left(e_{\rho}(y)\right), \\
l_{4}(\boldsymbol{u}, \boldsymbol{h}, \rho)=v(\boldsymbol{N} \cdot S(\boldsymbol{u}) \boldsymbol{N}-\boldsymbol{n} \cdot \widetilde{S}(\boldsymbol{u}) \boldsymbol{n})-\left[\boldsymbol{n}\left(e_{\rho}\right) \cdot T_{M}\left(\frac{\mathcal{L}}{J} \boldsymbol{h}\right) \boldsymbol{n}\right], \\
l_{5}(\rho)=-\sigma \int_{0}^{1}(1-s) \frac{d^{2}}{d s^{2}} \mathcal{L}^{-T}(y, s \rho) \nabla \cdot \frac{\widetilde{\mathcal{L}}^{T}(y, s \rho) \boldsymbol{N}}{\left|\widetilde{\mathcal{L}}^{T}(y, s \rho) \boldsymbol{N}\right|} d s, \\
l_{6}(\boldsymbol{u}, \boldsymbol{h}, \rho)=\left(\frac{\widehat{\mathcal{L}}^{T} \boldsymbol{N}}{\Lambda(y, \rho)}+\nabla_{\tau} \rho-\boldsymbol{N}\right) \cdot \boldsymbol{u}+(\boldsymbol{V}-\boldsymbol{u}) \cdot \nabla_{\tau} \rho, \quad y \in \mathcal{G}, \\
\boldsymbol{l}_{7}(\boldsymbol{h}, \rho)=\alpha^{-1}(\operatorname{roth}-\mathcal{P} r o t \mathcal{P})+\mu_{1}\left(\mathcal{L}^{-1} \boldsymbol{u} \times \boldsymbol{h}\right), \quad y \in \mathcal{F}_{1}, \\
\boldsymbol{l}_{8}(\boldsymbol{h}, \rho)=(I-\mathbb{P}) \boldsymbol{h}, \quad y \in \mathcal{F}_{2}, \\
\boldsymbol{l}_{9}(\boldsymbol{h}, \rho)=\left(\frac{\widehat{\mathcal{L}} \widehat{\mathcal{L}}^{T} \boldsymbol{N}}{\left|\widehat{\mathcal{L}}^{T} \boldsymbol{N}\right|^{2}}-\boldsymbol{N}\right)[\boldsymbol{h} \cdot \boldsymbol{N}]=[\boldsymbol{A}(\boldsymbol{h}, \rho)], \quad y \in \mathcal{G}, \\
\boldsymbol{A}^{(i)}(\boldsymbol{h}, \rho)=\left(\frac{\widehat{\mathcal{L}}^{T} \widehat{N}^{*}}{\left|\widehat{\mathcal{L}}^{T} \boldsymbol{N}^{*}\right|^{2}}-\boldsymbol{N}^{*}\right)\left(\boldsymbol{h}^{(i)} \cdot \boldsymbol{N}^{*}\right), \quad y \in \mathcal{F}_{i}, \quad i=1,2, \\
\Pi \boldsymbol{f}=\boldsymbol{f}-\boldsymbol{n}(\boldsymbol{n} \cdot \boldsymbol{f}), \quad \Pi_{\mathrm{g}} \boldsymbol{g}=\boldsymbol{g}-\boldsymbol{N}(\boldsymbol{g} \cdot \boldsymbol{N}) .
\end{array}\right.
$$

We note that the vector field

$$
\boldsymbol{\Phi}(\boldsymbol{h}, \rho)=\frac{1}{J} \widehat{\mathcal{L}}_{t} \mathcal{L} \boldsymbol{h}+\rho_{t}^{*} \widehat{\mathcal{L}}\left(\mathcal{L}^{-1} N^{*}(y) \cdot \nabla\right) \frac{1}{J} \mathcal{L} \boldsymbol{h}=\boldsymbol{h}_{t}(y, t)-\widehat{\mathcal{L}}\left(\left.\boldsymbol{H}_{t}(x, t)\right|_{x=e_{\rho}}\right)
$$

is divergence free and $\boldsymbol{\Phi}=0$ in $\mathcal{F}_{3}$ and in the neighborhood of $S \cup S_{3}$. 
The solvability of the problem (4.5) can be proved by successive approximations, according to a usual scheme

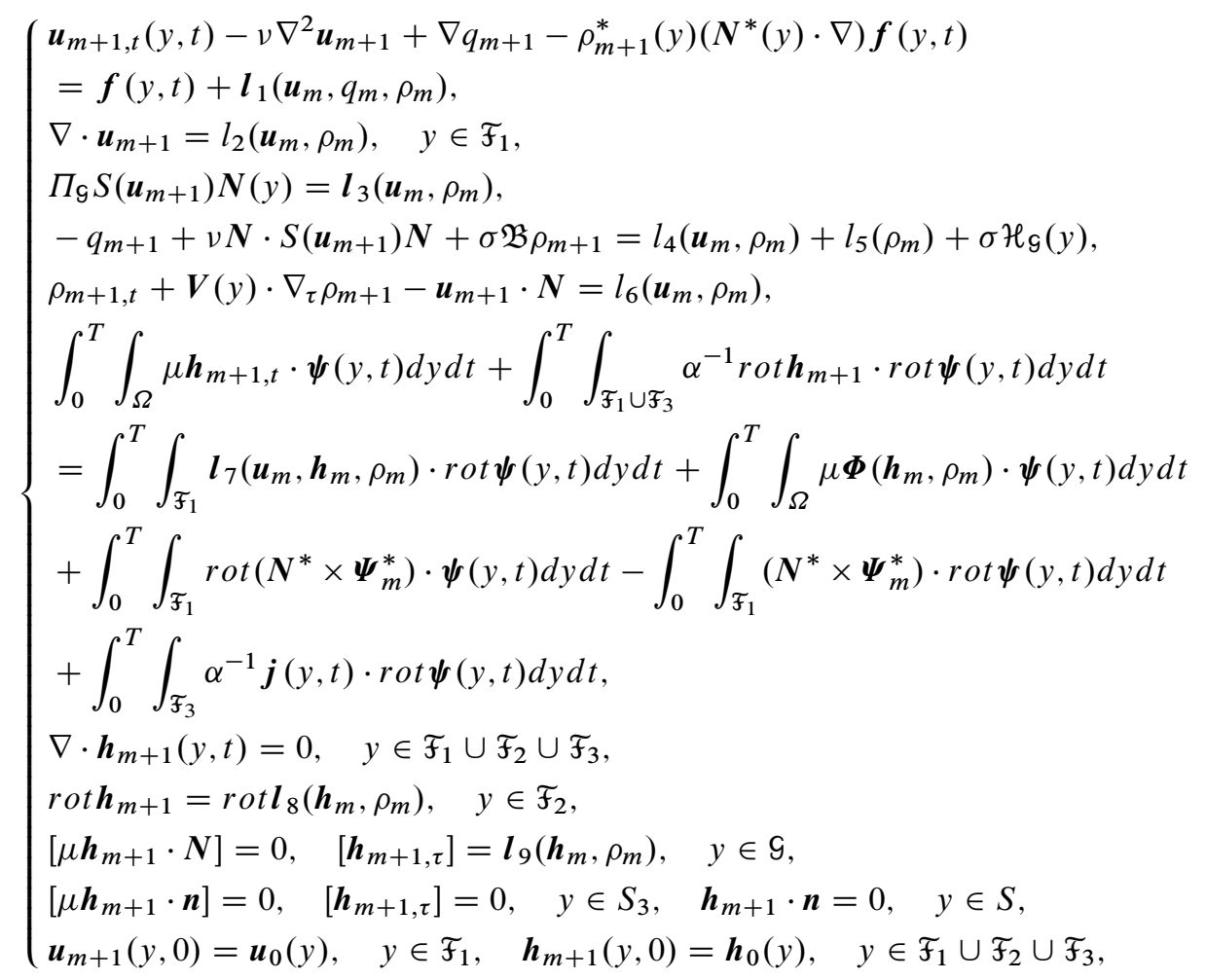

where $m=1, \ldots$, and $\boldsymbol{\Psi}_{m}^{*}$ is defined by (4.3) with $\boldsymbol{u}_{m}, \boldsymbol{h}_{m}, \rho_{m}$ instead of $\boldsymbol{u}, \boldsymbol{h}, \rho$.

The first approximation, $\left(\boldsymbol{u}_{1}, q_{1}, \rho_{1}, \boldsymbol{h}_{1}\right)$, is defined for $t \in(0, \infty)$ in the following way: $q_{1}=0$, $\boldsymbol{u}_{1}, \rho_{1}$ and $\boldsymbol{h}_{1}$ satisfy the initial conditions

$$
\begin{array}{ll}
\boldsymbol{u}_{1}(y, 0)=\boldsymbol{u}_{0}(y), & y \in \mathcal{F}_{1}, \quad \rho_{1}(y, 0)=\rho_{0}(y), \quad y \in \mathcal{G}, \\
\boldsymbol{h}_{1}(y, 0)=\boldsymbol{h}_{0}(y), \quad y \in \mathcal{F}_{1} \cup \mathcal{F}_{2} \cup \mathcal{F}_{3},
\end{array}
$$

the equation $\nabla \cdot \boldsymbol{h}_{1}(y, t)=0, y \in \mathcal{F}_{1} \cup \mathcal{F}_{2} \cup \mathcal{F}_{3}$ and the inequalities

$$
\begin{aligned}
& \left\|\boldsymbol{u}_{1}\right\|_{H^{l+2, l / 2+1}\left(Q_{\infty}^{1}\right)} \leqslant c\left\|\boldsymbol{u}_{1}\right\|_{W_{2}^{l+2, l / 2+1}\left(Q_{\infty}^{1}\right)} \leqslant c\left\|\boldsymbol{u}_{0}\right\|_{W_{2}^{l+1}\left(\mathcal{F}_{1}\right)}, \\
& \left\|\rho_{1}\right\|_{W_{2}^{l+5 / 2,0}\left(G_{\infty}\right)}+\left\|\rho_{1, t}\right\|_{W_{2}^{l+3 / 2, l / 2+3 / 4}\left(G_{\infty}\right)} \leqslant c\left\|\rho_{0}\right\|_{W_{2}^{l+2}(\mathcal{G})}, \\
& \sum_{i=1}^{3}\left\|\boldsymbol{h}_{1}\right\|_{W_{2}^{l^{\prime}+2, l^{\prime} / 2+1}\left(Q_{\infty}^{i}\right)} \leqslant c \sum_{i=1}^{3}\left\|\boldsymbol{h}_{0}\right\|_{W_{2}^{l^{\prime}+1}\left(\mathcal{F}_{i}\right)}
\end{aligned}
$$

(concerning the construction of $\rho_{1}$, see [23], Proposition 4.1). The proof of the solvability of (4.7) is based on the estimates of non-linear terms and of the solutions of the linear Problems 1 and 2 
studied in Sec. 3. Let

$$
\begin{aligned}
X_{m}(T)=\left\|\boldsymbol{u}_{m}\right\|_{H^{l+2, l / 2+1}\left(Q_{T}^{1}\right)}+ & \left\|\nabla q_{m}\right\|_{\widehat{W}_{2}^{l, l / 2}\left(Q_{T}^{1}\right)}+\left\|q_{m}\right\|_{W_{2}^{l+1 / 2,0}\left(G_{T}\right)} \\
& +\left\|q_{m}\right\|_{\widehat{W}_{2}^{l / 2}\left(0, T ; W_{2}^{1 / 2}(\mathcal{G})\right)} \\
+\left\|\rho_{m}\right\|_{W_{2}^{l+5 / 2,0}\left(G_{T}\right)}+ & \left\|\rho_{m}\right\|_{\widehat{W}_{2}^{l / 2}\left(0, T ; W_{2}^{5 / 2}(\mathcal{G})\right)}+\left\|\rho_{m, t}\right\|_{H^{l+3 / 2, l / 2+3 / 4}\left(G_{T}\right)} \\
& +\sup _{t<T}\left\|\rho_{m}(\cdot, t)\right\|_{W_{2}^{l+2}(\mathcal{G})}+\sum_{i=1}^{3}\left\|\boldsymbol{h}_{m}^{(i)}\right\|_{H^{l^{\prime}+2, l^{\prime} / 2+1}\left(Q_{T}^{i}\right)}
\end{aligned}
$$

and

$$
\begin{aligned}
& Z_{m}(T)=\left\|\boldsymbol{l}_{1}\left(\boldsymbol{u}_{m}, q_{m}, \boldsymbol{h}_{m}, \rho\right)\right\|_{\widehat{W}_{2}^{l, l / 2}\left(Q_{T}^{1}\right)}+\left\|l_{2}\left(\boldsymbol{u}_{m}, \rho_{m}\right)\right\|_{W_{2}^{l+1,0}\left(Q_{T}^{1}\right)}+\sup _{t<T}\left\|l_{2}\left(\boldsymbol{u}_{m}, \rho_{m}\right)\right\|_{W_{2}^{l}\left(\mathfrak{F}_{1}\right)} \\
& +\left\|\boldsymbol{L}_{t}\left(\boldsymbol{u}_{m}, \rho_{m}\right)\right\|_{\widehat{W}_{2}^{0, l / 2}\left(Q_{T}^{1}\right)}+\left\|\boldsymbol{l}_{3}\left(\boldsymbol{u}_{m}, \rho_{m}\right)\right\|_{H^{l+1 / 2, l / 2+1 / 4}\left(G_{T}\right)}+\left\|l_{4}\left(\boldsymbol{u}_{m}, \boldsymbol{h}_{m}, \rho_{m}\right)\right\|_{W_{2}^{l+1 / 2,0}\left(G_{T}\right)} \\
& +\left\|l_{5}\left(\rho_{m}\right)\right\|_{W_{2}^{l+1 / 2,0}\left(G_{T}\right)}+\left\|l_{4}\left(\boldsymbol{u}_{m}, \boldsymbol{h}_{m}, \rho_{m}\right)\right\|_{\widehat{W}_{2}^{l / 2}\left(0, T ; W_{2}^{1 / 2}(\mathcal{G})\right)}+\left\|l_{5}\left(\rho_{m}\right)\right\|_{\widehat{W}_{2}^{l / 2}\left(0, T ; W_{2}^{1 / 2}(G)\right)} \\
& +\left\|l_{6}\left(\boldsymbol{u}_{m}, \rho_{m}\right)\right\|_{H^{l+3 / 2, l / 2+3 / 4}\left(G_{T}\right)}+\left\|\boldsymbol{l}_{7}\right\|_{W_{2}^{l^{\prime}+1,0}\left(Q_{T}^{1}\right)}+\left\|\boldsymbol{l}_{7}\right\|_{\widehat{W}_{2}^{l^{\prime} / 2}\left(0, T ; W_{2}^{1}\left(\mathcal{F}_{1}\right)\right)} \\
& +\left\|\boldsymbol{l}_{8}\right\|_{H^{l^{\prime}+2, l^{\prime} / 2+1}\left(Q_{T}^{2}\right)}+\left\|\boldsymbol{l}_{9}\left(\boldsymbol{h}_{m}, \rho_{m}\right)\right\|_{H^{l^{\prime}+3 / 2, l^{\prime} / 2+3 / 4}\left(G_{T}\right)}+\left\|\boldsymbol{A}\left(\boldsymbol{h}_{m}, \rho_{m}\right)\right\|_{\widehat{W}_{2}^{0,1+l^{\prime} / 2}\left(Q_{T}\right)} \\
& +\sum_{i=1}^{3}\left\|\boldsymbol{\Phi}\left(\boldsymbol{h}_{m}, \rho_{m}\right)\right\|_{\widehat{W}_{2}^{l^{\prime}, l^{\prime} / 2}\left(Q_{T}^{i}\right)} \\
& +\left\|\boldsymbol{\Psi}^{*}\left(\boldsymbol{u}_{m}, \boldsymbol{h}_{m}, \rho_{m}\right)\right\|_{W_{2}^{l^{\prime}+1,0}\left(Q_{T}^{1}\right)}+\left\|\boldsymbol{\Psi}^{*}\left(\boldsymbol{u}_{m}, \boldsymbol{h}_{m}, \rho_{m}\right)\right\|_{\widehat{W}_{2}^{l^{\prime} / 2}\left(0, T ; W_{2}^{1}\left(\mathcal{F}_{1}\right)\right)} .
\end{aligned}
$$

The following proposition is an analog of Theorem 7 in [20].

Proposition 2 Assume that (4.4) holds and

$$
\sup _{t<T}\left\|\rho_{m}(\cdot, t)\right\|_{W_{2}^{l+3 / 2}(\mathcal{G})} \leqslant \delta \ll 1,
$$

moreover, let the extension $\rho^{*}$ of $\rho$ (see (2.2)) possess the properties

$$
\left.\frac{\partial \rho^{*}}{\partial N}\right|_{g}=0,
$$

$\rho^{*}(y, t)=0$, when $y \in \mathcal{F}_{3}$ or $y$ belongs to a neighborhood of $S$ and

$$
\begin{aligned}
& \left\|\rho^{*}(\cdot, t)\right\|_{W_{2}^{r+1 / 2}(\Omega)} \leqslant c\|\rho\|_{W_{2}^{r}(\mathcal{G})}, \quad r \in(0, l+5 / 2], \\
& \left\|\rho_{t}^{*}(\cdot, t)\right\|_{W_{2}^{r+1 / 2}(\Omega)} \leqslant c\left\|\rho_{t}\right\|_{W_{2}^{r}(\mathcal{G})}, \quad r \in(0, l+3 / 2], \quad l \in(1 / 2,1) .
\end{aligned}
$$

Then

$$
Z_{m} \leqslant \delta_{1} \sum_{j=1}^{3} X_{m}^{j},
$$

where $\delta_{1}$ is a small number dependent on $\delta$ and $T$. 
The non-linear terms (4.6) are identical or very similar to the nonlinear terms (1.10), (1.11) in [20], and they are estimated in the same manner (cf. [20], Sec. 4, [22], Sec. 3). We point out that the estimate of $\boldsymbol{l}_{9}$ are made here slightly better than in [20], because the norms in $W_{2}^{-1 / 2}(G)$ are excluded from the final inequality (4.12).

Making use of the inequalities (3.6) and (3.31) applied to the problem (4.7), we obtain

$$
X_{m+1} \leqslant c_{1} \delta_{1} \sum_{j=1}^{3} X_{m}^{j}+c_{2} N
$$

where $\delta_{1} \ll 1$,

$$
\begin{aligned}
N=\left\|\boldsymbol{u}_{0}\right\|_{W_{2}^{l+1}\left(\mathcal{F}_{1}\right)}+\left\|\rho_{0}\right\|_{W_{2}^{l+2}(\mathcal{G})}+\left\|\mathcal{H}_{g}\right\|_{W_{2}^{l+1 / 2}(\mathcal{G})} & \\
& +\sum_{i=1}^{3}\left\|\boldsymbol{h}_{0}\right\|_{W_{2}^{l^{\prime}+1}\left(\mathcal{F}_{i}\right)}+\|\boldsymbol{j}\|_{W_{2}^{l^{\prime}+1,0}\left(Q_{T}^{3}\right)}+\|\boldsymbol{j}\|_{\widehat{W}_{2}^{l^{\prime} / 2}\left(0, T ; W_{2}^{1}\left(\mathcal{F}_{3}\right)\right)}+\|\boldsymbol{f}\|_{\widehat{W}_{2}^{l, l / 2}\left(Q_{T}^{1}\right)}
\end{aligned}
$$

and the constants $c_{1}$ and $c_{2}$ are independent of $T$.

It follows that in the case of small $\delta_{1}$ the estimate

$$
X_{m+1} \leqslant 2 c_{2} N
$$

holds and, in addition,

$$
\begin{aligned}
& \left\|\rho_{m}(\cdot, t)\right\|_{W_{2}^{l+3 / 2}(\mathcal{G})} \leqslant\left\|\rho_{0}\right\|_{W_{2}^{l+3 / 2}(\mathcal{G})}+\int_{0}^{t}\left\|\rho_{m, \tau}(\cdot, \tau)\right\|_{W_{2}^{l+3 / 2}(\mathcal{G})} d \tau \\
& \leqslant\left\|\rho_{0}\right\|_{W_{2}^{l+3 / 2}(\mathrm{~g})}+2 c_{2} N \sqrt{T} \leqslant \epsilon+2 c_{2} \sqrt{T} N \equiv \delta .
\end{aligned}
$$

Thus, we have proved that (4.13) is satisfied for all $m \geqslant 1$. The convergence of the sequences $\boldsymbol{u}_{m}$, $q_{m}, \boldsymbol{h}_{m}, \rho_{m}$ follows from the estimates of the differences $\boldsymbol{u}_{m+1}-\boldsymbol{u}_{m}, q_{m+1}-q_{m}, \boldsymbol{h}_{m+1}-\boldsymbol{h}_{m}$, $\rho_{m+1}-\rho_{m}$ (cf. [20], Theorem 8). Making $m$ tend to infinity, we obtain the inequality

$$
X(T) \leqslant 2 c_{2} N
$$

for the solution of the problem (4.7) constructed above ( here $X(T)$ is defined by (4.9) with $\boldsymbol{u}, q, \rho, \boldsymbol{h}$ instead of $\boldsymbol{u}_{m}, q_{m}, \rho_{m}, \boldsymbol{h}_{m}$ ).

The uniqueness of the solution obtained in this way follows from the same kind of estimates for the differences $\boldsymbol{u}-\boldsymbol{u}^{\prime}, q-q^{\prime}, \rho-\rho^{\prime}, \boldsymbol{h}-\boldsymbol{h}^{\prime}$ of two possible solutions of (4.5).

We conclude the proof of Theorem 1 by the construction of the vector field $\boldsymbol{e}$ assuming that the solution of (4.1) is already obtained. For this we need to solve the problem

$$
\left\{\begin{array}{l}
\operatorname{rot} \mathcal{E}(y)=\mu \xi(y), \quad \nabla \cdot \mathbb{P}^{-1} \mathcal{E}=0, \quad y \in \mathcal{F}_{1} \cup \mathcal{F}_{2} \cup \mathcal{F}_{3}, \\
{\left[\mu \mathcal{P}^{-1} \mathcal{E} \cdot \boldsymbol{N}\right]=0, \quad\left[\mathcal{E}_{\tau}\right]=\boldsymbol{\alpha}(y), \quad y \in \mathcal{G},} \\
{\left[\mu \mathcal{P}^{-1} \mathcal{E} \cdot \boldsymbol{n}\right]=0, \quad\left[\mathcal{E}_{\tau}\right]=0, \quad y \in S_{3}, \quad \mathcal{E}_{\tau}=0, \quad y \in S .}
\end{array}\right.
$$


THEOREM 6 Assume that $\xi \in W_{2}^{l^{\prime}}\left(\mathcal{F}_{i}\right), i=1,2,3, \boldsymbol{\alpha} \in W_{2}^{l^{\prime}+1 / 2}(\mathcal{G})$ and that the compatibility and orthogonality conditions

$$
\begin{aligned}
& \nabla \cdot \boldsymbol{\xi}(y)=0, \quad y \in \mathcal{F}_{1} \cup \mathcal{F}_{2} \cup \mathcal{F}_{3}, \\
& {[\mu \boldsymbol{\xi} \cdot \boldsymbol{N}]=\boldsymbol{N} \cdot \operatorname{rot} \boldsymbol{\alpha}, \quad y \in \mathcal{G}, \quad[\mu \boldsymbol{\xi} \cdot \boldsymbol{n}]=0, \quad y \in S_{3}, \quad \boldsymbol{\xi} \cdot \boldsymbol{n}=0, \quad y \in S,} \\
& \int_{\Omega} \mu \boldsymbol{\xi}(y) \cdot \widetilde{\boldsymbol{u}}_{q}(y) d y=\int_{\mathcal{G}}(\boldsymbol{N} \times \boldsymbol{\alpha}) \cdot \widetilde{\boldsymbol{u}}_{q} d S, \quad \widetilde{\boldsymbol{u}}_{q} \in \widetilde{U}_{n}(\Omega), \quad q=1, \ldots, b_{1}(\Omega)
\end{aligned}
$$

are satisfied. Then the problem (4.16) has a unique solution $\varepsilon \in W_{2}^{l^{\prime}+1}\left(\mathcal{F}_{i}\right), i=1,2,3$ orthogonal to the $b_{2}(\Omega)$-dimensional space $\widetilde{U}_{d}$ of vector fields $v(y)$ such that

$$
\begin{aligned}
& \operatorname{rot} \boldsymbol{v}=0, \quad \nabla \cdot \mathbb{P}^{-1} \boldsymbol{v}(y)=0, \\
& {\left[\boldsymbol{v}_{\tau}\right]=0, \quad y \in S_{3} \cup \mathcal{G},\left.\quad\left[\mu \mathcal{P}^{-1} \boldsymbol{v} \cdot \boldsymbol{N}\right]\right|_{\mathcal{G}}=0,\left.\quad\left[\mu \mathcal{P}^{-1} \boldsymbol{v} \cdot \boldsymbol{n}\right]\right|_{S_{3}}=0,\left.\quad \boldsymbol{v}_{\tau}\right|_{S}=0 .}
\end{aligned}
$$

The solution satisfies the inequality

$$
\sum_{i=1}^{3}\|\varepsilon\|_{W_{2}^{r+1}\left(\mathcal{F}_{i}\right)} \leqslant c\left(\sum_{i=1}^{3}\|\xi\|_{W_{2}^{r}\left(\mathcal{F}_{i}\right)}+\|\boldsymbol{\alpha}\|_{W_{2}^{r+1 / 2}(\mathcal{G})}\right)
$$

with $r=l^{\prime}, r=0$.

Proof. We seek the solution in the form

$$
\varepsilon=\boldsymbol{\alpha}^{*}+\varepsilon_{1}+\nabla Z(y)+\sum_{j=1}^{b_{2}(\Omega)} d_{j} \boldsymbol{v}_{j}(y),
$$

where $\boldsymbol{\alpha}^{*}$ is the extension of $\boldsymbol{\alpha}$, as in Theorem 3,

$$
\begin{gathered}
\mathcal{E}_{1}(y)=\frac{1}{4 \pi} \operatorname{rot} \int_{\Omega} \frac{\left(\mu \xi(z)-\operatorname{rot} \boldsymbol{\alpha}^{*}(z)\right) d z}{|y-z|}, \\
\left\{\begin{array}{l}
\nabla \cdot \mathbb{P}^{-1} \nabla Z=-\nabla \mathbb{P}^{-1}\left(\boldsymbol{\alpha}^{*}+\mathcal{E}_{1}(y)\right), \quad y \in \mathcal{F}_{1} \cup \mathcal{F}_{2} \cup \mathcal{F}_{3}, \\
Z(y)=-g(y), \quad y \in S, \quad[Z(y)]=0, \quad y \in \mathcal{G} \cup S_{3}, \\
{\left[\mu \mathbb{P}^{-1} \nabla Z \cdot N\right]=-\left[\mu \mathbb{P}^{-1}\left(\boldsymbol{\alpha}^{*}+\mathcal{E}_{1}\right) \cdot \boldsymbol{N}\right], \quad y \in \mathcal{G},} \\
{\left[\mu \mathbb{P}^{-1} \nabla Z \cdot \boldsymbol{n}\right]=-\left[\mu \mathbb{P}^{-1}\left(\boldsymbol{\alpha}^{*}+\mathcal{E}_{1}\right) \cdot \boldsymbol{n}\right], \quad y \in S_{3} .}
\end{array}\right.
\end{gathered}
$$

It is easily seen that $\operatorname{rot} \varepsilon_{1}=\mu \xi-\operatorname{rot} \alpha^{*}$. The function $g$ is defined as follows. Since $(\mu \xi-$ $\left.\operatorname{rot} \boldsymbol{\alpha}^{*}\right)\left.\cdot \boldsymbol{n}\right|_{S}=0$, we have $\int_{\Sigma} \operatorname{rot} \varepsilon_{1} \cdot \boldsymbol{n} d S=0$ for arbitrary $\Sigma \subset S$, and this relation holds also for $\Sigma=\Sigma_{k}, k=1, \ldots, b_{1}(\Omega)$, where $\Sigma_{k}$ are cuts of $\Omega$ that make $\Omega$ simply connected (this follows from the orthogonality conditions (4.18), i.e., from

$$
\int_{\Omega}\left(\mu \boldsymbol{\xi}-\operatorname{rota}^{*}\right) \cdot \boldsymbol{u}_{q} d y=0
$$

see [6]). Hence by the Stokes formula, the relation

$$
\int_{\gamma} \varepsilon_{1} \cdot d \boldsymbol{l}=0
$$


holds for arbitrary closed contour $\gamma \subset S$, which implies $\left.\varepsilon_{1}\right|_{S}=\nabla_{\tau} g(y)$ with a certain singlevalued $g(y)$. Now the relations (4.16) are easily verified.

Making use of the estimates of the volume potential and of the solution of elliptic problem (4.22) for $Z$, we estimate $\varepsilon_{1}$ and $\nabla Z$, after which the term $\sum_{j=1}^{b_{2}(\Omega)} d_{j} \boldsymbol{v}_{j}(y)$ is estimated with the help of the condition of orthogonality of $\varepsilon$ to $v_{j}$ (cf. the proof of (3.15)). In this way we obtain (4.20). The uniqueness of the solution in the class indicated in the statement of the theorem is obvious. The theorem is proved.

Now we pass to the construction of $\boldsymbol{e}$. We want to solve the problem (4.16) with $\boldsymbol{\xi}=-\boldsymbol{h}_{t}+\boldsymbol{\phi}$ and $\boldsymbol{\alpha}=-\boldsymbol{N} \times \boldsymbol{\Psi}$; in this case the condition $\left[\varepsilon_{\tau}\right]=\boldsymbol{\alpha}$ on $\mathcal{G}$ is equivalent to

$$
[N \times \varepsilon]=\Psi .
$$

Let us verify the assumptions (4.17), (4.18) of Theorem 6. From Proposition 1 it follows that arbitrary $\boldsymbol{b}$ and $\mathcal{E}$ given on $\mathcal{F}_{1} \cup \mathcal{F}_{2}$ and $\mathcal{G}$, respectively, and such that

$$
\begin{aligned}
& \nabla \cdot \boldsymbol{b}=0, \quad y \in \mathcal{F}_{1} \cup \mathcal{F}_{2}, \quad[\boldsymbol{b} \cdot \boldsymbol{N}]=0, \quad y \in \mathcal{G}, \\
& {[\boldsymbol{N} \times \mathcal{E}]=\frac{\boldsymbol{u} \cdot \widehat{\mathcal{L}}^{T} \boldsymbol{N}}{J}[\boldsymbol{b}], \quad y \in \mathcal{G},}
\end{aligned}
$$

satisfy

$$
[\boldsymbol{N} \cdot \operatorname{rot} \mathcal{E}]=-\left[\boldsymbol{N} \cdot\left(\boldsymbol{b}_{t}-\frac{1}{L} \widehat{\mathcal{L}}^{T} \mathcal{L} \boldsymbol{b}-\rho_{t} \widehat{\mathcal{L}}\left(\mathcal{L}^{-1}(\boldsymbol{N} \cdot \nabla) \frac{1}{L} \mathcal{L} \boldsymbol{b}\right)\right] .\right.
$$

For $\boldsymbol{b}=\mu \boldsymbol{h}$, this coincides with the main compatibility condition in (4.17). As for (4.18), this condition can be verified by setting $\boldsymbol{\psi}=\widetilde{\boldsymbol{u}}_{q}$ in the integral identity in (4.1). Hence the problem (4.16) with the above-mentioned data is solvable, and the solution satisfies the identity

$$
\begin{aligned}
& \int_{0}^{T} \int_{\mathcal{F}_{1} \cup \mathcal{F}_{3}}\left(-\varepsilon+\alpha^{-1} \operatorname{roth}\right) \cdot \operatorname{rot} \psi(y, t) d y d t \\
& =\int_{0}^{T} \int_{\mathfrak{F}_{1}} \boldsymbol{l}_{7}(\boldsymbol{u}, \boldsymbol{h}, \rho) \cdot \operatorname{rot} \boldsymbol{\psi}(y, t) d y d t+\int_{0}^{T} \int_{\mathfrak{F}_{3}} \alpha^{-1} \boldsymbol{j}(y, t) \cdot \operatorname{rot} \boldsymbol{\psi}(y, t) d y d t .
\end{aligned}
$$

By Theorem 3 , rot $\psi$ runs over the whole $\stackrel{\circ}{J}\left(\mathfrak{F}_{1} \cup \mathfrak{F}_{3}\right)$, when $\psi$ runs over $\mathcal{H}^{1}(\Omega)$. It follows that

$$
\begin{aligned}
& -\varepsilon^{(1)}+\alpha_{1}^{-1} \operatorname{ProtP} \boldsymbol{h}^{(1)}-\mu_{1}\left(\mathcal{L}^{-1} \boldsymbol{u} \times \boldsymbol{h}^{(1)}\right)=\nabla Y^{(1)}, \\
& -\varepsilon^{(3)}+\alpha^{-1} \operatorname{roth}^{(3)}-\alpha^{-1} \boldsymbol{j}(y, t)=\nabla Y^{(3)} .
\end{aligned}
$$

We set

$$
\mathcal{P} \boldsymbol{e}=\mathcal{E}+\nabla Y, \quad y \in \mathfrak{F}_{1} \cup \mathcal{F}_{3}
$$

and

$$
\boldsymbol{P} \boldsymbol{e}=\varepsilon+\nabla Y^{(2)}+\sum_{j=1}^{b_{2}(\Omega)} C_{j}(t) \nabla \omega_{j}(y), \quad y \in \mathcal{F}_{2},
$$

where $Y^{(2)}$ is a solution of the problem

$$
\nabla \cdot \mathbb{P}^{-1} \nabla Y^{(2)}=0, \quad y \in \mathcal{F}_{2},\left.\quad Y^{(2)}\right|_{y \in \mathcal{G}}=Y^{(1)},\left.\quad Y^{(2)}\right|_{y \in S_{3}}=Y^{(3)},\left.\quad Y^{(2)}\right|_{y \in S}=0
$$


and $\omega_{j}$ are solutions of

$$
\nabla \cdot \mathbb{P}^{-1} \nabla \omega_{j}=0, \quad y \in \mathcal{F}_{2},\left.\quad \omega_{j}(y)\right|_{y \in S_{k}}=\delta_{j k}, \quad j, k=1, \ldots, b_{2}\left(\mathcal{F}_{2}\right),\left.\quad \omega_{j}\right|_{y \in \mathcal{S}}=0 .
$$

Then the equations

$$
\begin{gathered}
\nabla \cdot \boldsymbol{e}^{(2)}(y, t)=0, \quad y \in \mathcal{F}_{2},\left.\quad\left[(\mathbb{P} \boldsymbol{e})_{\tau}\right]\right|_{y \in \mathcal{G}}=\boldsymbol{\alpha}, \quad y \in \mathcal{G} \\
{\left.\left[(\mathbb{P} \boldsymbol{e})_{\tau}\right]\right|_{y \in S_{3}}=0,\left.\quad(\mathbb{P} \boldsymbol{e})_{\tau}\right|_{y \in S}=0}
\end{gathered}
$$

are satisfied.

We pass to the estimates of $\boldsymbol{e}$. By (4.20),

$$
\sum_{i=1}^{3}\|\varepsilon\|_{W_{2}^{l^{\prime}+1,0}\left(Q_{T}^{i}\right)} \leqslant c\left(\sum_{i=1}^{3}\left\|\boldsymbol{h}_{t}-\Phi\right\|_{W_{2}^{l^{\prime}, 0}\left(Q_{T}^{i}\right)}+\|\boldsymbol{\Psi}\|_{W_{2}^{l^{\prime}+1 / 2,0}\left(G_{T}\right)}\right) .
$$

Applying the same inequality with $l^{\prime}=0$ to $\varepsilon^{\prime}$ and to the finite difference $\Delta_{t}(h) \mathcal{E}=\varepsilon(y, t+h)-$ $\varepsilon^{\prime}(y, t)$, we easily obtain

$$
\sum_{i=1}^{3}\|\varepsilon\|_{\widehat{W}_{2}^{l^{\prime} / 2}\left(0, T ; W_{2}^{1}\left(\mathcal{F}_{i}\right)\right)} \leqslant c\left(\sum_{i=1}^{3}\left\|\boldsymbol{h}_{t}-\Phi\right\|_{\widehat{W}_{2}^{0, l^{\prime} / 2}\left(Q_{T}^{i}\right)}+\|\boldsymbol{\Psi}\|_{\widehat{W}_{2}^{l^{\prime} / 2}\left(0, T ; W_{2}^{1 / 2}(\mathcal{G})\right.}\right) .
$$

Hence

$$
\begin{aligned}
& \sum_{i=1}^{3}\left(\|\varepsilon\|_{W_{2}^{l^{\prime}+1,0}\left(Q_{T}^{i}\right)}+\|\varepsilon\|_{\widehat{W}_{2}^{l^{\prime} / 2}\left(0, T ; W_{2}^{1}\left(\mathcal{F}_{i}\right)\right)}\right) \\
& \leqslant c\left(\sum_{i=1}^{3}\left\|\boldsymbol{h}_{t}-\boldsymbol{\Phi}\right\|_{\widehat{W}_{2}^{l^{\prime}, l^{\prime} / 2}\left(Q_{T}^{i}\right)}+\|\boldsymbol{\Psi}\|_{W_{2}^{l^{\prime}+1 / 2,0}\left(G_{T}\right)}+\|\boldsymbol{\Psi}\|_{\widehat{W}_{2}^{l^{\prime} / 2}\left(0, T ; W_{2}^{1 / 2}(\mathcal{G})\right.}\right) .
\end{aligned}
$$

Furthermore, from (4.25) it follows that

$$
\begin{aligned}
& \left\|\nabla Y^{(1)}\right\|_{W_{2}^{l^{\prime}+1,0}\left(Q_{T}^{1}\right)} \leqslant\|\varepsilon\|_{W_{2}^{l^{\prime}+1,0}\left(Q_{T}^{1}\right)} \\
& +\left\|\alpha_{1}^{-1} \operatorname{Prot} \operatorname{Ph}-\mu_{1}\left(\mathcal{L}^{-1} \boldsymbol{u} \times \boldsymbol{h}\right)\right\|_{W_{2}^{l^{\prime}+1,0}\left(Q_{T}^{1}\right)}, \\
& \left\|\nabla Y^{(1)}\right\|_{\widehat{W}_{2}^{l^{\prime} / 2}\left(0, T ; W_{2}^{1}\left(\mathcal{F}_{1}\right)\right)} \leqslant\|\varepsilon\|_{\widehat{W}_{2}^{l^{\prime} / 2}\left(0, T ; W_{2}^{1}\left(\mathcal{F}_{1}\right)\right)} \\
& +\left\|\alpha_{1}^{-1} \operatorname{Prot} \boldsymbol{P} \boldsymbol{h}-\mu_{1}\left(\mathcal{L}^{-1} \boldsymbol{u} \times \boldsymbol{h}\right)\right\|_{\widehat{W}_{2}^{l^{\prime} / 2}\left(0, T ; W_{2}^{1}\left(\mathcal{F}_{1}\right)\right)}, \\
& \left\|\nabla Y^{(3)}\right\|_{W_{2}^{l^{\prime}+1,0}\left(Q_{T}^{1}\right)} \leqslant\|\varepsilon\|_{W_{2}^{l^{\prime}+1,0}\left(Q_{T}^{3}\right)}+\left\|\alpha^{-1}(\operatorname{roth}-\boldsymbol{j})\right\|_{W_{2}^{l^{\prime}+1,0}\left(Q_{T}^{1}\right)}, \\
& \left\|\nabla Y^{(3)}\right\|_{\widehat{W}_{2}^{l^{\prime} / 2}\left(0, T ; W_{2}^{1}\left(\mathcal{F}_{3}\right)\right)} \leqslant\|\varepsilon\|_{\widehat{W}_{2}^{l^{\prime} / 2}\left(0, T ; W_{2}^{1}\left(\mathcal{F}_{3}\right)\right)}+\left\|\alpha^{-1}(\operatorname{rot} \boldsymbol{h}-\boldsymbol{j})\right\|_{\widehat{W}_{2}^{l^{\prime} / 2}\left(0, T ; W_{2}^{1}\left(\mathcal{F}_{3}\right)\right)} \text {. }
\end{aligned}
$$

In addition, since $Y^{(2)}$ is a solution of (4.26), we have

$$
\begin{aligned}
&\left\|\nabla Y^{(2)}\right\|_{W_{2}^{l^{\prime}+1,0}\left(Q_{T}^{2}\right)}+\left\|\nabla Y^{(2)}\right\|_{\widehat{W}_{2}^{l^{\prime} / 2}\left(0, T ; W_{2}^{1}\left(\mathfrak{F}_{2}\right)\right)} \\
& \leqslant c\left(\left\|\nabla Y^{(1)}\right\|_{W_{2}^{l^{\prime}+1,0}\left(Q_{T}^{1}\right)}+\left\|\nabla Y^{(1)}\right\|_{\widehat{W}_{2}^{l^{\prime} / 2}\left(0, T ; W_{2}^{1}\left(\mathcal{F}_{1}\right)\right)}\right. \\
&\left.\quad+\left\|\nabla Y^{(3)}\right\|_{W_{2}^{l^{\prime}+1,0}\left(Q_{T}^{3}\right)}+\left\|\nabla Y^{(3)}\right\|_{\widehat{W}_{2}^{l^{\prime} / 2}\left(0, T ; W_{2}^{1}\left(\mathcal{F}_{3}\right)\right)}\right) .
\end{aligned}
$$


The functions $C_{j}(t)$ are found from the condition (1.13), since the matrix with the elements $\int_{S_{k}} \mathbb{P}^{-1} \nabla \omega_{j} \cdot \boldsymbol{n} d S$ is non-degenerate. Indeed, otherwise there would exist the constants $a_{j}$ such that

$$
\int_{S_{k}} \mathbb{P}^{-1} \nabla A(y) \cdot \boldsymbol{n} d S=0, \quad k=1, \ldots, b_{2}(\Omega)
$$

where $A(y)=\sum_{j=1}^{b_{2}(\Omega)} a_{j} \omega_{j}$. This would imply

$$
\begin{aligned}
0 & =\int_{\Omega} A \nabla \cdot \mathbb{P}^{-1} \nabla A d y=\int_{\partial \mathcal{F}_{2}} \mathbb{P}^{-1} \nabla A(y) \cdot \boldsymbol{n} A(y) d S-\int_{\mathcal{F}_{2}} \mathbb{P}^{-1} \nabla A(y) \cdot \nabla A(y) d y \\
& =-\int_{\mathcal{F}_{2}} \mathcal{P}^{-1} \nabla A(y) \cdot \nabla A(y) d y,
\end{aligned}
$$

because $\left.A\right|_{S_{k}}=$ const, $k=0, \ldots, b_{2}\left(\mathcal{F}_{2}\right)$. Hence $\nabla A=0$ and $a_{j}=0$.

By (1.13),

$$
\begin{aligned}
0=\int_{S_{k}} \boldsymbol{e} \cdot \boldsymbol{n} d S=\int_{S_{k}} \boldsymbol{P} \boldsymbol{e} \cdot \boldsymbol{n} d S=\int_{S_{k}}\left(\varepsilon+\nabla Y^{(2)}\right) \cdot \boldsymbol{n} d S & \\
& +\sum_{j=1}^{b_{2}(\Omega)} C_{j}(t) \int_{S_{k}} \nabla \omega_{j} \cdot \boldsymbol{n} d S,
\end{aligned}
$$

from which it follows that

$$
\begin{aligned}
& \left\|\sum_{j=1}^{b_{2}} C_{j} \mathbb{P}^{-1} \nabla \omega_{j}\right\|_{W_{2}^{l^{\prime}+1,0}\left(Q_{T}^{2}\right)}+\left\|\sum_{j=1}^{b_{2}} C_{j} \mathbb{P}^{-1} \omega_{j}\right\|_{\widehat{W}_{2}^{l^{\prime} / 2}\left(0, T ; W_{2}^{1}\left(\mathcal{F}_{2}\right)\right)} \leqslant c\left(\left\|\nabla Y^{(2)}\right\|_{W_{2}^{l^{\prime}+1,0}\left(Q_{T}^{2}\right)}\right. \\
& \left.+\left\|\nabla Y^{(2)}\right\|_{\widehat{W}_{2}^{l^{\prime} / 2}\left(0, T ; W_{2}^{1}\left(\mathcal{F}_{2}\right)\right)}+\|\varepsilon\|_{W_{2}^{l^{\prime}+1,0}\left(Q_{T}^{2}\right)}+\|\varepsilon\|_{\widehat{W}_{2}^{l^{\prime} / 2}\left(0, T ; W_{2}^{1}\left(\mathcal{F}_{2}\right)\right)}\right) .
\end{aligned}
$$

Finally,

$$
\begin{aligned}
& \sum_{i=1}^{3}\left(\|\boldsymbol{e}\|_{W_{2}^{l^{\prime}+1,0}\left(Q_{T}^{i}\right)}+\|\boldsymbol{e}\|_{\widehat{W}_{2}^{l^{\prime} / 2}\left(0, T ; W_{2}^{1}\left(\mathcal{F}_{i}\right)\right)}\right) \leqslant c \sum_{i=1}^{3}\left(\|\mathcal{E}\|_{W_{2}^{l^{\prime}+1,0}\left(Q_{T}^{i}\right)}\right. \\
& +\|\mathcal{E}\|_{\widehat{W}_{2}^{l^{\prime} / 2}\left(0, T ; W_{2}^{1}\left(\mathcal{F}_{i}\right)\right)}+\left\|\nabla Y^{(i)}\right\|_{W_{2}^{l^{\prime}+1,0}\left(Q_{T}^{i}\right)}+\left\|\nabla Y^{(i)}\right\|_{\widehat{W}_{2}^{l^{\prime} / 2}\left(0, T ; W_{2}^{1}\left(\mathcal{F}_{i}\right)\right)} \\
& \left.+\left\|\sum_{j=1}^{b_{2}} C_{j} \mathbb{P}^{-1} \nabla \omega_{j}\right\|_{W_{2}^{l^{\prime}+1,0}\left(Q_{T}^{2}\right)}+\left\|\sum_{j=1}^{b_{2}} C_{j} \mathbb{P}^{-1} \omega_{j}\right\|_{\widehat{W}_{2}^{l^{\prime} / 2}\left(0, T ; W_{2}^{1}\left(\mathcal{F}_{2}\right)\right)}\right) .
\end{aligned}
$$

Inequality (2.19) for $\boldsymbol{e}$ is a consequence of (4.27)-(4.34), (4.12), (4.13). This completes the proof of Theorem 1.

The solution of the problem (1.2)-(1.5), (1.7), (1.12), (1.13) is defined by

$$
\begin{aligned}
& \boldsymbol{v}(x, t)=\boldsymbol{u}\left(e_{\rho}^{-1}(x, t), t\right), \quad p(x, t)=q\left(e_{\rho}^{-1}(x, t), t\right), \\
& \boldsymbol{H}(x, t)=\left.\frac{\mathcal{L}\left(y, \rho^{*}\right)}{J\left(y, \rho^{*}\right)} \boldsymbol{h}(x, t)\right|_{y=e_{\rho}^{-1}(x, t)}, \quad \boldsymbol{E}(x, t)=\left.\frac{\mathcal{L}\left(y, \rho^{*}\right)}{J\left(y, \rho^{*}\right)} \boldsymbol{e}(y, t)\right|_{y=e_{\rho}^{-1}(x, t)},
\end{aligned}
$$


the free surface $\Gamma_{t}$ is given by (2.1).

In conclusion we show that $\boldsymbol{h} \in W_{2}^{l+2, l / 2+1}\left(Q_{T}^{i}\right)$, provided $\boldsymbol{h}_{0} \in W_{2}^{l+1}\left(\mathcal{F}_{i}\right), i=1,2,3, \boldsymbol{j} \in$ $W_{2}^{l+1,0}\left(Q_{T}^{3}\right) \cap W_{2}^{l / 2}\left(0, T ; W_{2}^{1}\left(\mathcal{F}_{3}\right)\right)$ The condition $l^{\prime}<l$ was used for obtaining the estimate (4.17) with a small $\delta_{1}$ that is a linear combination of $\delta$ and $T_{i}^{\gamma}, \gamma_{i}>0$; in particular, $\gamma$ may be equal to $\left(l-l^{\prime}\right) / 2$. Analysis of such estimates (see [20], Sec 4. ) shows that the factor $T^{\left(l-l^{\prime}\right) / 2}$ appears in the estimates of the norms $\|(I-P) \boldsymbol{h}\|_{W_{2}^{l^{\prime}+2, l^{\prime} / 2+1}\left(Q_{T}^{i}\right)}$; they contain the expression

$$
\begin{aligned}
& c\left(\|\rho\|_{W_{2}^{5 / 2+l^{\prime}, 0}\left(G_{T}\right)}+\right.\left.\|\rho\|_{\widehat{W}_{2}^{l^{\prime} / 2}\left(0, T ;\left(W_{2}^{5 / 2}(\mathcal{G})\right)\right.}\right) \sum_{i=1}^{3} \sup _{t<T}\|\boldsymbol{h}\|_{\left.W_{2}^{3 / 2+n}\left(\mathcal{F}_{i}\right)\right)} \\
& \leqslant c T^{\left(l-l^{\prime}\right) / 2} \sup _{t<T}\|\rho(\cdot, t)\|_{W_{2}^{3 / 2+l}(\mathcal{G})}^{l-l^{\prime}}\left(\|\rho\|_{W_{2}^{l+5 / 2,0}\left(G_{T}\right)}^{1-l+l^{\prime}}\right. \\
&+\|\rho\|_{\widehat{W}_{2}^{l / 2}\left(0, T ; W_{2}^{5 / 2}\left(G_{T}\right)\right)}^{1-l+l^{\prime}} \sum_{i=1}^{3} \sup _{t<T}\|\boldsymbol{h}\|_{\left.W_{2}^{3 / 2+n}\left(\mathcal{F}_{i}\right)\right)}
\end{aligned}
$$

with $3 / 2+\eta<2+l^{\prime}$. In the estimate of $\|(I-\mathcal{P}) \boldsymbol{h}\|_{W_{2}^{l+2, l / 2+1}\left(Q_{T}^{i}\right)}$ this expression would be replaced by

$$
\begin{aligned}
& c\left(\|\rho\|_{W_{2}^{5 / 2+l, 0}\left(G_{T}\right)}+\|\rho\|_{W_{2}^{l / 2}\left(0, T ; W_{2}^{5 / 2}(\mathcal{G})\right)}\right) \sum_{i=1}^{3} \sup _{t<T}\|\boldsymbol{h}\|_{\left.W_{2}^{3 / 2+n}\left(\mathcal{F}_{i}\right)\right)} \\
& \quad \leqslant c\left(\|\rho\|_{W_{2}^{5 / 2+l, 0}\left(G_{T}\right)}+\|\rho\|_{\widehat{W}_{2}^{l / 2}\left(0, T ; W_{2}^{5 / 2}(\mathcal{G})\right)}\right) \sum_{i=1}^{3} \sup _{t<T}\|\boldsymbol{h}\|_{\left.W_{2}^{2+l^{\prime}, 1+l^{\prime} / 2}\left(Q_{T}^{i}\right)\right)},
\end{aligned}
$$

that is finite in view of (2.19). This yields the missing bound for the norm

$$
\sum_{i=1}^{3}\|\boldsymbol{h}\|_{\left.W_{2}^{2+l, 1+l / 2}\left(Q_{T}^{i}\right)\right)} .
$$

It is easily seen that also

$$
\boldsymbol{e} \in W_{2}^{l+1,0}\left(Q_{T}^{i}\right) \cap W_{2}^{l / 2}\left(0, T ; W_{2}^{1}\left(\mathcal{F}_{i}\right)\right),
$$

which follows from the estimates (4.27)-(4.34) with $l^{\prime}$ replaced by $l$.

Acknowledgments. The work is supported by the research grant 11-01-00324 of RFBR.

\section{REFERENCES}

1. LAndau, L.D. \& Lifshitz, E.M., Electrodynamics of continuouis media, Pergamon Press (1960). Zbl0122.45002 MR0121049

2. Roberts, P. H., An introduction in magnetohydrodynamics, London, Longmans, Green and Co. LTD (1967).

3. Ladyzhenskaya, \& O. A. Solonnikov, V. A., Solution of some non-stationary problems of magnetohydrodynamics for a viscous incompressible fluid, Proc. Mat. Inst. Steklov, 59 (1960), 115-173. 
4. Ladyzhenskaya, O. A. \& Solonnikov, V. A., The linearization principle and invariant manifolds for problems of magnetohydrodynamics, J. of Math. Sci. 8 (1977), 384-422. Zbl0404. 35089

5. Sanchez Palincia, E., Quelques résultats d'existence et d'unicité pour des ecoulements magnetohydrodynamiques non stationnaires, J. méc. 8 (1969), 501-541. Zb10193.27301

6. LASSner, G., Ueber ein Rand-Anfangswertproblem der Magnetohydrodynamik, Arch. Rat. Mech Anal. 25 (1967), 388-405. Zbl0152.45502 MR0216809

7. Duvaut, G. \& Lions, J. L., Inequations en Thermoelasticité et Magnetohydrodynamique, Arch. Ration. Mech. Anal. 46 (1972), 241-279. Zbl0264.73027 MR0346289

8. SAKHAEV, SH. \& Solonnikov, V. A., Estimates of solutions of a boundary value problem of magnetohydrodynamics, Trudy Mat. Inst. Steklov 127, (1975), 76-92. Zb10366.76091

9. SAKHAEv, Sh., Estimate of the solution of an overdetermined parabolic initial-boundary value problem, Trudy Mat. Inst. Steklov 127, (1975), 58-75.

10. Giga, Y. \& Yoshida, Z., On the Ohm-Navier-Stokes system in magnetohydrodynamics, J. Math. Phys. 24 (1983), 2860-2864. Zbl0533.76125 MR0727780

11. Sermange, M. \& Temam, R., Some mathematical questions related to the MHD equations, Comm. Pure Appl. Math. 36 (1983), 635-664. Zb10524.76099 MR0716200

12. Mulone, G. \& Solonnikov, V. A., On the solvability of some initial-boundary value problems of magnetohydrodynamics with Hall and ion-slip effects, Atti Acad. Naz. Lincei 6 (1995), 117-132. Zb10834.76094 MR1354225

13. Ströhmer, G., About an initial-boundary value problem from magnetohydrodynamics, Math. Z. 209 (1992), 345-362. Zbl0756.76095 MR1152262

14. StröHMER, G., An existence result for partially regular weak solutions of certain abstract evolution equations, with an application to magnetohydrodynamics, Math. Z. 213 (1993), 373-385. Zb10806. 35057 MR1227488

15. Ströhmer, G., About the equations of non-stationary magnetohydrodynamics with non-conducting boundaries, Nonlin. Anal. 39 (2000), 629-647. Zb10959.76095 MR1727268

16. Ströhmer, G. About the equations of non-stationary magnetohydrodynamics, Nonlin. Anal. 52 (2003), 1249-1273. Zbl1021.35088 MR1941256

17. Mosconi, S. \& Solonnikov, V. A., On a problem of magnetohydrodynamics in a multi-connected domain, Nonlinear Analysis 74 (2010), 462-478. Zblpre05819590 MR2733223

18. Kiselev, A.A. \& Ladyzhenskaya, O. A., On the existence and uniqueness of the solution of the nonstationary problem for a viscous incompressible fluid, Izv. Acad. Nauk SSSR, Ser. Mat. 21 (1957), 655-680. Zb10131.41201

19. LAdYZHENSKAYA, O.A., Solution "in the large" of the nonstationary boundary value problem for the Navier-Stokes system with two space variables, Comm. Pure Appl. Math 12 (1959), 427-433. Zbl0103. 19502

20. Padula, M. \& Solonnikov, V. A., On the free boundary problem of magnetohydrodynamics, J. Math. Sci. 178 (2011), 313-344. MR2749373

21. HanZawA, EI-ICHI, Classical solutions of the Stefan problem, Tohoku Math. J. 33 (1981), 297-335. Zb10571.35109 MR0633045

22. Padula, M. \& Solonnikov, V. A., On the local solvability of free boundary problem for the NavierStokes equations, J. Math. Sci. 170 No 4 (2010), 522-553. MR2839878

23. Solonnikov, V. A., On the linear problem arising in the study of free boundary problem for the NavierStokes equations, St. Petersburg Math. J. 22 (2011), 1023-1049. Zbl1230. 35090 MR2760093

24. Bykhovskit, E. B. \& Smirnov, N. V., On the orthogonal decomposition of the space of vector functions square summable in a given domain and the operators of vector analysis, Tr. Mat. Inst. Steklov 59 (1960), 6-36. MR0121641

25. WEYL, H., The method of orthogonal decomposition in potential theory, Duke Math. J. 7 (1940), 411-444. Zb10026.02001 MR0003331 